\title{
Semiclassical theory of molecular spectral line shapes in gases*
}

\author{
D. E. Fitz and R. A. Marcus \\ Department of Chemistry, University of Illinois, Urbana, Illinois 61801
}

(Received 28 June 1973)

\begin{abstract}
A semiclassical theory of the width and shift of molecular spectral lines is developed for gases. Overlapping and nonoverlapping lines are considered, within the framework of the impact approximation. Use is made of "exact" semiclassical theory of molecular collisions, recently developed by Miller and by Marcus, and of developments in the quantum mechanical theory of spectral line shapes, by introducing the former into the latter. Comparison is made with a classical-like approach.
\end{abstract}

\section{INTRODUCTION}

There have been a number of recent developments, both in the theory of collisional line broadening ${ }^{1-14}$ and in the "exact" semiclassical theo$\mathrm{ry}^{15,16}$ of collisions. In the present paper, these two trends are blended to yield a semiclassical theory of spectral line broadening, valid within the impact approximation and specifically for the case of foreign gas broadening for overlapping and nonoverlapping lines.

The advances in the theory of line broadening based on the "impact approximation" (an approximation which allows the collisions of perturbers with the absorbing molecule to be treated as well separated in time), include the pioneering work of Anderson, ${ }^{1}$ who employed a classical path approximation (classical plus only zeroth order treatment of the relative motion between perturber and absorber) and a perturbative approximation for the $S$-matrices appearing in the line shape expression. This classical path result was later extended to overlapping lines by Baranger ${ }^{2}$ and Kolb and Griem, ${ }^{3}$ and by Gersten and Foley, ${ }^{4}$ who also made detailed calculations for actual systems. Rabitz, Gordon, and Nielson ${ }^{5,6}$ made further developments in these calculations. An all-quantum-mechanical formal treatment was developed by Baranger ${ }^{7}$ to replace the classical path approximation and the use of perturbative expansions of the $S$-matrices. This formalism was made more compact with the introduction of Liouville space ("line space") formalism by Fano. ${ }^{8}$ The latter was further developed by Ben-Reuven, ${ }^{9}$ who introduced a practical treatment for the high-orbital-rotational degeneracy present and proceeded to treat several specific overlapping line systems.

No $a b$ initio calculations for this "exact" (within the impact approximation) formalism appear to have been published. However, there have been many $a b$ initio calculations using the classical path, mostly with ${ }^{10}$ but some without ${ }^{6}$ perturbative schemes for the internal motions. A nonperturbative classical-like model has been described by
Gordon ${ }^{11}$ and has been used recently by Gordon and co-workers ${ }^{6,12}$ to calculate various line shapes for overlapping and nonoverlapping systems. Recent related reviews of relaxation phenomena rele vant to line broadening have been given by Gordon, Klemperer, and Steinfeld ${ }^{13}$ and by Beenakker, Knaap, and Sanctuary. ${ }^{14}$

The quantum mechanical formalism for the line shape $^{8,9}$ is first converted in Sec. II into a form, which contains "collision cross sections" $\sigma_{i^{\prime} f^{\prime} ; i f}$. The "exact" semiclassical expressions recently developed by one of $\mathrm{us}^{15}$ and by Miller ${ }^{16}$ for the $S$ matrices ${ }^{17}$ are then introduced into the crosssection and a semiclassical expression for the Wigner $6-j$ symbols $^{18}$ is also employed. Both of these appear in the expression for the line shape which is valid within the impact approximation. This expression is developed first for a linear molecule perturbed by a foreign gas in Sec. III. The extension of these expressions to related relaxation phenomena ${ }^{13}$ is described in Sec. IV [Eqs. (4. 1) and (4.4)]. The latter phenomena will also be the subject of further papers of this series.

\section{QUANTUM MECHANICAL LINE SHAPE EXPRESSION}

The assumptions made in each of the all-quantum-mechanical treatments for foreign gas broadening cited earlier, and used here to obtain a tractable line shape expression valid within the impact approximation, are as follows: (i) the absorber and perturber distribution functions are essentially uncorrelated, (ii) the system is dilute enough in absorber molecules that absorber-absorber interactions are minor and may be neglected, (iii) it is at low enough pressures that the approximation of binary absorber-perturber molecular collisions may be used, and (iv) the impact approximation is valid.

The spectral line shape $I(\omega)$ for electric dipole transitions is then given for overlapping lines by $^{9,19}$

$$
I(\omega)=-(1 / \pi) \operatorname{Im} \sum_{i i^{\prime} f f^{\prime}}\left\langle f^{\prime}\|\mu\| \cdot i^{\prime}\right\rangle
$$




$$
\begin{aligned}
& \times\left\langle\left\langle i^{\prime} f^{\prime} ;-1,1,0\left|1 /\left[\omega-\mathbf{L}_{0}^{a}-N\{\mathrm{~m}(\omega)\}\right]\right|\right.\right. \\
& \times \text { if } ;-1,1,0\rangle\rangle \rho_{i}\langle i\|\mu\| f\rangle,
\end{aligned}
$$

where Im denotes imaginary part; $i$ and $f$ refer to states of the absorber (emitter) before and after the optical transition, respectively; unprimed $i$ and $f$ denote such absorber states before collision and primed quantities such states after collision; $\rho_{i}$ is the probability of finding the absorber (emitter) in state $i$; the $-1,1$, and 0 describe the parity ( -1 for an electric dipole transition) and the relevant tensorial properties of interaction with the radiation; $\omega$ is the frequency of the absorbed (emitted) radiation and, on the right hand side of (2.1), is written as positive for emission and negative for absorption; ${ }^{2} \mathrm{~L}_{0}^{a}$ is the Liouville operator for the unperturbed absorber; $N$ is the foreign gas density (perturbers); $\mu$ is the electric dipole operator; and $\{\mathrm{m}(\omega)\}$ is the binary collision Liouville operator, averaged over all perturber states:

$$
\{\mathbf{m}(\omega)\}=\sum_{\zeta \zeta^{\prime}} \rho_{\zeta}\left\langle\left\langle\zeta^{\prime} \zeta^{\prime}|m(\omega)| \zeta \zeta\right\rangle\right\rangle,
$$

where $\zeta$ and $\zeta^{\prime}$ denote the state of perturber (including the translational state of absorber-perturber relative motion) before and after collision, respectively; $\rho_{\xi}$ is the probability of finding this perturber in initial state $\zeta$. When the effects of any internal states of the perturber are neglected, as with monatomic inert gas perturbers at the usual temperatures $\left(300^{\circ} \mathrm{K}\right.$, for example), $\zeta$ denotes only the translational state. We set $\hbar=1$ throughout. For completeness, notation and operations ${ }^{9,20}$ for the Liouville operator $L_{0}^{a}$, the reduced dipole matrix elements and the (doublebracketted) Liouville vectors in (2.1) and (2.2) are summarized in Appendix A.

The operator relationship ${ }^{8}$ between the binary collision operator $m(\omega)$ and the transition operator of scattering theory can be converted to reduced matrix form. ${ }^{9 b}$ The details and subsequent conversion to $S$-matrices and collision cross sections are summarized in Appendix B. One obtains

$$
\begin{array}{r}
\left\langle\left\langle i^{\prime} f^{\prime} ;-1,1,0|\{m(\omega)\}| i f ;-1,1,0\right\rangle\right\rangle \\
=-i \int_{0}^{\infty} v \sigma_{i f^{\prime} ; i f} \rho_{v} 4 \pi v^{2} d v,
\end{array}
$$

where $\rho_{v} 4 \pi v^{2} d v$ is the normalized Maxwell-Boltzmann distribution and $\sigma_{i \prime f^{\prime} ; \text { if }}$ is a cross section for a collision of an absorber-perturber pair

$$
\begin{aligned}
& \sigma_{i \prime f^{\prime} ; i f}=\left(\pi / k^{2}\right) \sum_{l t^{\prime} J_{i} J_{f}}(-)^{j_{i}-j_{i}^{\prime}+l-l^{\prime}}\left(2 J_{i}+1\right)\left(2 J_{f}+1\right) \\
& \times\left\{\begin{array}{ccc}
J_{f} & J_{i} & 1 \\
j_{i} & j_{f} & l
\end{array}\right\}\left\{\begin{array}{c}
J_{f} J_{i} 1 \\
j_{i}^{\prime} j_{f}^{\prime} l^{\prime}
\end{array}\right\}\left[\delta_{1^{\prime} 1} \delta_{f^{\prime},}-S_{1^{\prime},}^{J} S_{f^{\prime} \mathrm{f}}^{J_{f}^{\prime}}\right] .
\end{aligned}
$$

Here, each $j$ denotes the absorber's rotational angular momentum quantum number; $i$ and $f$ de- note properties of the absorber (emitter) before and after an optical transition, respectively; the $i$ and $f$ subscripts for $\sigma$ denote $\pi_{\mathfrak{i}} j_{i}$ and $\pi_{f} j_{f}$ for pure rotational lines and $\pi_{i} j_{i} n_{i}$ and $\pi_{f} j_{f} n_{f}$ for rotational-vibrational lines $\left(n_{i} \pi_{i}\right.$ and $n_{f} \pi_{f}$ are the vibrational quantum numbers and parity before and after the optical transition, respectively); unprimed and primed symbols refer to pre- and postcollision quantities; $l$ denotes the orbital angular momentum quantum number $\left(l=l_{i}=l_{f}, l^{\prime}\right.$ $\left.=l_{i}^{\prime}=l_{f}^{\prime}\right) ; J$ denotes the total angular momentum quantum number (e.g., $J_{i}=j_{i}+l=j_{i}^{\prime}+l^{\prime}$ ); and the $i$ and $\mathbf{f}$ denote $\pi_{i} j_{i} l$ and $\pi_{f} j_{f} l$, respectively for pure rotational lines, and denote $\pi_{i} n_{i} j_{i} l$ and $\pi_{f} n_{f} j_{f} l$, respectively for rotational-vibrational lines; $k$ is $\mu v / \hbar$, i.e., $\mu v$; the distribution function $\rho_{v}$ is

$$
\rho_{v}=\left(\mu / 2 \pi k_{B} T\right)^{3 / 2} \exp \left(-\mu v^{2} / 2 k_{B} T\right),
$$

$k_{B}$ being Boltzmann's constant, $T$ the temperature of the system, and $\mu$ the reduced mass of the absorber-perturber pair [not to be confused with the $\mu$ in (2.1)]. The $S^{J_{i}}$ and $S^{J_{f}}$ matrix elements in (2.4) are evaluated at different energies, e.g., as in Eq. (B19), Appendix B. Equation (2.4) is valid within the impact approximation (cf. Ref. 7).

Knowing the matrix elements of $N\{\mathbf{m}(\omega)\}$ in (2.3), those of $\mathbf{L}_{0}^{a}\left(=E_{i}^{0}-E_{f}^{0}\right)$ and of $\omega\left(=\omega \delta_{i f}\right)$, the matrix elements of $\left[\omega-\mathrm{L}_{0}^{a}-N\{\mathrm{~m}(\omega)\}\right]^{-1}$ in (2.1) can be cal culated by a suitable inversion method.

Equation (2.4) is converted to semiclassical form in Sec. III.

\section{SEMICLASSICAL LINE SHAPE EXPRESSION}

In this section, an expression for the line shape is developed, but the variables describing vibration are excluded for brevity. These variables pose no difficulty and are included in the final expression Eq. (3.21) for overlapping lines at the end of this section.

A semiclassical expression for the $6-j$ symbols, appropriate for the case of two large and one small angular momenta, is ${ }^{18}$

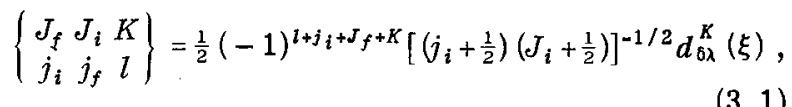

where $\lambda=J_{f}-J_{i}, \delta=j_{f}-j_{i}(\lambda, \delta= \pm 1,0)$, and $\xi$ is the angle between the vectors $\mathrm{j}_{i}$ and $\mathrm{J}_{i}$,

$\cos \xi=\left[\left(J_{i}+\frac{1}{2}\right)^{2}+\left(j_{i}+\frac{1}{2}\right)^{2}-\left(l+\frac{1}{2}\right)^{2}\right] / 2\left(j_{i}+\frac{1}{2}\right)\left(J_{i}+\frac{1}{2}\right)$.

A similar expression applies to the other $6-j$ symbol.

In semiclassical theory ${ }^{15,16}$ the states $i$ and $f$ are most conveniently described in terms of action-angle variables. The action corresponding 
to a quantum number $n$ is $(n+\delta) h$ where $\delta$ [not to be confused with the $\delta$ in (3.1)] is, depending on the degree of freedom, frequently $\frac{1}{2}$ or, in the case of $z$ components of angular momenta, 0 . In units of $\hbar=1$, this action variable becomes $2 \pi(n+\delta)$. The coordinate canonically conjugate to the action is the angle variable $w$, which varies from 0 to 1 . However, $w$ is also canonically conjugate to $2 \pi n$ in the appropriate sense of satisfying the correct Poisson-bracket relation for canonically conjugate quantities. $^{21}$

A collision of an atom and a (rigid) linear molecule can be described in the center of mass system with the aid of the initial action variables $2 \pi\left(l+\frac{1}{2}\right), 2 \pi\left(j+\frac{1}{2}\right)$, and $2 \pi\left(J+\frac{1}{2}\right)$, the initial radial momentum $p_{R}$, and their canonically conjugate coordinates, $w_{l}, w_{j}, w_{J}$, and $R$. The dynamics of the collision are unaffected by the one remaining action variable $2 \pi M$, which is the $z$ component of $\mathbf{J}$, and by its canonically conjugate coordinate $w_{M}$. Several of these $w$ 's, multiplied by $2 \pi$ and called $q$ 's, are shown in Fig. 1. For convenience, we have set $\mathbf{J}$ along a space-fixed $z$ axis, and so will not exhibit the (presently unneeded) angle $2 \pi w_{M}$. The angle $2 \pi w_{j}$ is measured in the $\mathrm{J}$ plane (plane perpendicular to $J$ ) in the direction indicated from the space-fixed $Y$ axis to the line of intersection of the $\mathrm{j}$ and 1 planes, "the line of nodes $O N . "$ The angle $2_{\pi} w_{j}$ is measured in the $\mathrm{j}$-plane from $O N$ to the position of the dipole $O D$. Similarly, the angle $2 \pi w_{l}$ is measured in the 1 -plane from $O N$ to the position of the line of centers $O C$ of the collision partners. The postcollision coordinates $w_{l}^{\prime}, w_{j}^{\prime}$, $w_{J}^{\prime}$, and $R^{\prime}$ are canonically conjugate to the postcollision momenta, $2 \pi\left(l^{\prime}+\frac{1}{2}\right), 2 \pi\left(j^{\prime}+\frac{1}{2}\right), 2 \pi\left(J^{\prime}+\frac{1}{2}\right)$, and $p_{R}^{\prime}$. The angles are the same as those in Fig. 1 when primes are placed on the symbols in that figure.

Finally, in our previous semiclassical papers ${ }^{15}$ we used $\left(w^{0}, R^{0}\right)$ and $(w, R)$ to denote initial and final coordinates, respectively, and $\left(2 \pi n, p_{R}^{0}\right)$ and $\left(2 \pi \bar{n}, p_{R}\right)$ to denote their canonically conjugate vari-

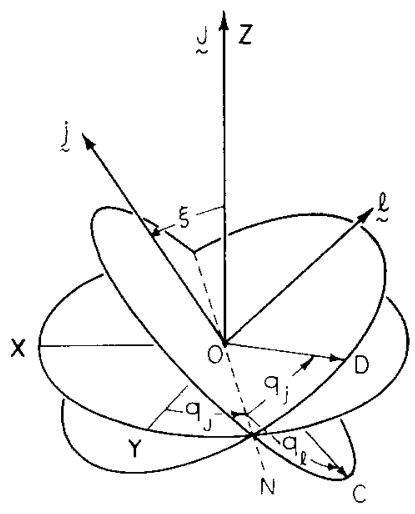

FIG. 1. Variables describing internal coordinates $q_{j}, q_{l}$, and $q_{J}$ for the motion of an atom and rigid rotor. The rotor axis lies along $O D$ and the line of centers of the collision partners lies along $O C$, at any instant of time. The $q$ 's are $2 \pi$ times the corresponding $w$ 's. ables. We have now modified the notation so that these quantities are now $(w, R),\left(w^{\prime}, R^{\prime}\right),\left(2 \pi n, p_{R}\right)$, and $\left(2 \pi n^{\prime}, p_{R}^{\prime}\right)$, respectively, to conform with a common line broadening practice of using unprimed and primed variables to represent pre- and postcollision quantities.

The semiclassical expression for the $S$-matrix can be written as ${ }^{22}$

$$
\begin{aligned}
S_{j_{i} l^{\prime} ; j_{i} l}^{J_{i}}= & \sum\left|-i \partial\left(j_{i}^{\prime}, l^{\prime}\right) / \partial\left(w_{j_{i}}, w_{l}\right)\right|^{-1 / 2} \\
& \times \exp \left[i F_{4}\left(j_{i}^{\prime} l^{\prime} J_{i} E_{i} ; j_{i} l J_{i} E_{i}\right)+\frac{1}{2}\left(l+l^{\prime}+1\right) \pi\right] .
\end{aligned}
$$

$F_{4}$ is a classical mechanical generating function, ${ }^{23}$ for transforming from the precollision momenta $j_{i} l J_{i} p_{R}$ to the postcollision momenta $j_{i}^{\prime} l_{i}^{\prime} J_{i} p_{R}^{\prime}$ :

$$
\begin{aligned}
F_{4}\left(j_{i}^{\prime} l^{\prime} J_{i} E ; j_{i} l J_{i} E\right)= & -\int_{l}^{l^{\prime}} 2 \pi w w_{l} d l-\int_{j}^{j^{\prime}} 2 \pi w_{j} d j \\
& -\int_{P_{R}}^{P_{R}{ }^{\prime}} R d p_{R}
\end{aligned}
$$

where the integration in (3.4) is over the classical trajectory leading from the initial precollision state $j_{i} l J_{i} E$ to the desired postcollision final state $j_{i}^{\prime} l^{\prime} J_{i} E$. The summation in (3.3) is over all such trajectories (real or complex-valued) which lead from this initial state to this final state.

The exponent of $S^{J_{i}} S^{J_{f}^{*}}$ in $(2.4)$ can be written as

$$
\text { exponent in } \begin{aligned}
S_{f_{1}}^{J_{i}} S_{1 / 4}^{J_{f}} & =i F_{4}\left(j_{i}^{\prime} l^{\prime} J_{i}^{\prime}, E_{i}^{\prime} ; j_{i} l J_{i}, E_{i}\right) \\
& -i F_{4}^{*}\left(j_{f}^{\prime} l^{\prime} J_{f}^{\prime} E_{f}^{\prime} ; j_{f} l J_{f} E_{f}\right) .
\end{aligned}
$$

Each $F_{4}$ is real valued when the relevant trajectories are real valued.

When the $F_{4}$ and $F_{4}^{*}$ in (3.5) are expanded about a common value, retaining the first two terms of the expansion, we have

$$
\begin{aligned}
& F_{4}\left(j_{i}^{\prime} l^{\prime} J_{i}^{\prime}, E_{i}^{\prime} ; j_{i} l J_{i}, E_{i}\right)-F_{4}^{*}\left(j_{f}^{\prime} l^{\prime} J_{f}^{\prime}, E_{f}^{\prime} ; j_{f} l J_{f}, E_{f}\right) \\
& \cong 2 i \Phi+\frac{\partial F_{4}}{\partial j_{i}}\left(j_{i}-j_{f}\right)+\frac{\partial F_{4}}{\partial j_{i}^{\prime}}\left(j_{i}^{\prime}-j_{f}^{\prime}\right)+\frac{\partial F_{4}}{\partial J_{i}}\left(J_{i}-J_{f}\right) \\
&+\frac{\partial F_{4}}{\partial J_{i}^{\prime}}\left(J_{i}^{\prime}-J_{f}^{\prime}\right)+\frac{\partial F_{4}}{\partial E_{i}}\left(E_{i}-E_{f}\right)+\frac{\partial F_{4}}{\partial E_{i}^{\prime}}\left(E_{i}^{\prime}-E_{f}^{\prime}\right),
\end{aligned}
$$

where $\Phi=\operatorname{Im} F_{4}$. These derivatives and $\Phi$ are evaluated at a mean value $\left(j, j^{\prime}, J\right)$ of $\left(j_{i}, j_{i}^{\prime}, J_{i}\right)$ and $\left(j_{f}, j_{f}^{\prime}, J_{f}\right)$ and at a mean energy. We note that $J_{i}^{\prime}=J_{i}$ and $J_{f}^{\prime}=J_{f}$.

The partial derivatives in (3.6) are, respectively, $\bar{w}_{j},-\bar{w}_{j}^{\prime}, w_{J},-w_{j}^{\prime}, w_{E},-w_{E}^{\prime}$, as shown in Appendix C. Thereby, we have

$$
\text { lhs of }(3.6)=2 i \Phi+\theta_{1}+\theta_{2},
$$

where (Appendix C)

$$
\theta_{1}=2 \pi\left(\bar{w}_{j}^{\prime} \delta^{\prime}-\vec{w}_{j} \delta+w_{J}^{\prime} \lambda-w_{J} \lambda\right),
$$


$\theta_{2}=w_{E}\left(E_{i}-E_{f}\right)-w_{E}^{\prime}\left(E_{i}^{\prime}-E_{f}^{\prime}\right)$,

$w_{E}-w_{E}^{\prime}=t^{\prime}-t-\left(R^{\prime} / v^{\prime}\right)+(R / v),\left(v<0, v^{\prime}>0\right)$

$$
-\delta=j_{i}-j_{f},-\delta^{\prime}=j_{i}^{\prime}-j_{f}^{\prime},-\lambda=J_{i}-J_{f},
$$

and

$$
\bar{w}_{j}=w_{j}-R \nu_{j} / v, \bar{w}_{j}^{\prime}=w_{j}^{\prime}-R^{\prime} \nu_{j}^{\prime} / v^{\prime} .
$$

$\bar{w}_{j}, \bar{w}_{j}^{\prime}, w_{J}, w_{J}^{\prime}$ and $w_{E}^{\prime}-w_{E}$ are constants of the trajectory, independent of the initial $R$ and time and of the final values, $R^{\prime}$ and $t^{\prime} . \quad w_{E}^{\prime}-w_{E}$ is a "collision delay time," since it is the energy derivative of the phase of an $S$-matrix. ${ }^{24}$ The diagonal elements $i f=i^{\prime} f^{\prime}$ offer no difficulty and Eqs. (3.6) and (3.7) apply. For the off-diagonal elements one actually has off-the-energy shell $T$-matrix elements, ${ }^{7}$ as in Appendix C, rather than (2.4). The occurrence of these elements is also reflected in the fact that $E_{i}-E_{f}$ does not necessarily equal $E_{i}^{\prime}-E_{f}^{\prime}$ when if $\neq i^{\prime} f^{\prime}$, and hence that $E_{i} \neq E_{i}^{\prime}$ or $E_{f} \neq E_{f}^{\prime}$ in the $T$-matrix elements of line broadening. However, such differences between $E_{i}^{\prime}-E_{f}^{\prime}$ and $E_{i}-E_{f}$ are neglected, relative to $k T$, in the impact approximation. ${ }^{7}$ Within this approximation (2.4), (3.6), and (3.7) apply to the off-diagonal elements, if $\neq i^{\prime} f^{\prime}$, also.

We note, too, that one pre-exponential factor of $S^{S_{i}}$ in this expansion is, if we first consider the pre-exponential factor for the diagonal element $i f=i^{\prime} f^{\prime}$, equal to the complex conjugate of that of $S^{J_{f}}$. Neglecting interference terms, we now have

$$
\begin{aligned}
S_{j_{i}^{\prime} l^{\prime} ; j_{i} l}^{J_{i}} S_{j_{f}^{\prime} l^{\prime} ; j_{f} l}^{J_{f}} \cong & \sum\left|\partial\left(j^{\prime}, l^{\prime}\right) / \partial\left(\bar{w}_{f}, \bar{w}_{l}\right)\right|^{-1} \\
& \times \exp \left[i\left(\theta_{1}+\theta_{2}\right)-2 \Phi\right] .
\end{aligned}
$$

The semiclassical expression for the $\sigma_{i \prime f^{\prime} ; i f}$ in (2.4) becomes, with the aid of (3.1) and (3.11),

$$
\begin{aligned}
\sigma_{i, f^{\prime} ; i f}=\left(\pi / k^{2}\right) & \int_{0}^{\infty}(2 l+1) d l\left(\delta_{j_{i^{\prime} j_{i}}} \delta_{\tilde{f}_{f^{\prime} j_{f}}}-\sum \int_{0}^{\infty} d l^{\prime}\right. \\
& \times \int_{|j-l|}^{j+l} d J \frac{2 J+1}{(2 l+1) \sqrt{(2 j+1)\left(2 j^{\prime}+1\right)}} \\
& \times\left|\frac{\partial\left(j^{\prime}, l^{\prime}\right)}{\partial\left(\bar{w}_{j}, \bar{w}_{l}\right)}\right|^{-1} \exp \left(-2 \Phi+i \theta_{2}\right) D_{\delta^{\prime} \delta}^{1}(\alpha \beta \gamma),
\end{aligned}
$$

where $j$ and $J$ denote mean values of $\left(j_{i}, j_{f}\right)$ and

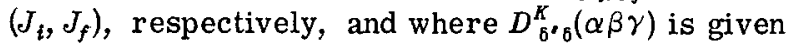
by $(3.13)$, with $K=1$;

$$
D_{6^{\prime}{ }_{b}}^{K}(\alpha \beta \gamma)=\sum_{\lambda=-K}^{K} e^{i \theta_{1}} d_{6^{\prime} \lambda}^{K}(\xi) d_{\sigma^{\prime} \lambda}^{K}\left(\xi^{\prime}\right) \text {. }
$$

The symbol $D_{6^{\prime} \delta}^{K}(\alpha \beta \gamma)$ defined by the right hand side of (3.13) proves to be the element of a rotation matrix, as shown in Appendix D, and describes the rotation of the "reduced" dipole $O \bar{D}$ into $O \bar{D}^{\prime}$ via the angles $\alpha, \beta$, and $\gamma$, as shown in Fig. 2. The angles $\alpha$ is the angle measured in the j-plane from the "reduced" dipole $O \bar{D}$ to the line

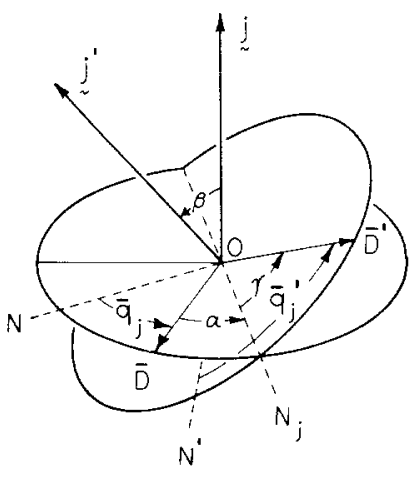

FIG. 2. Angles $\alpha, \beta, \gamma$ for rotating the "reduced dipole" $O \bar{D}$ into $O \bar{D}$ '. The $O N$ is the same as the $O N$ in Fig. 1, and $O N^{\prime}$ is the corresponding quantity for the primed variables. Each $\bar{q}$ denotes $2 \pi \bar{w}$, and $\bar{w}=w$ $-R \nu / v . \quad O N_{j}$ is the line of intersection of the rotational the one after. plane before collision with

of nodes $O N_{j}$ of the $\mathrm{j}$ and $\mathbf{j}^{\prime}$ planes. The angle $\beta$ is the angle of rotation about $O N_{j}$ from $j$ to $j^{\prime}$. The angle $\gamma$ is the angle measured in the $\mathbf{j}^{\prime}$ plane from $O N_{j}$ to the "reduced" dipole $O \bar{D}^{\prime}$. (Thus, $\alpha \beta \gamma$ describes the rotation of $O \bar{D}$ into $O \bar{D}^{\prime}$.) An angle measured from $O \bar{D}$ to the actual dipole $O D$ in the $\mathrm{j}$ plane can be seen from (3.10) to be $2 \pi R \nu_{j} / v$, while the corresponding angle from $O \bar{D}^{\prime}$ to $O D^{\prime}$ in the $\mathrm{j}^{\prime}$ plane is $2 \pi R^{\prime} \nu_{j}^{\prime} / v^{\prime} . \quad D_{0^{\prime}{ }_{0}}^{K}(\alpha \beta \gamma)$ is

$$
D_{6^{\circ}{ }_{6}}^{K}(\alpha \beta \gamma)=e^{i \sigma^{*} \alpha} d_{6^{\prime}{ }_{6}}^{K}(\beta) e^{i 6^{*} \gamma}
$$

following the convention in Edmonds. ${ }^{25}$

Inasmuch $\mathrm{as}^{26}$

$$
\left|\partial\left(j^{\prime}, l^{\prime}\right) / \partial\left(\bar{w}_{j}, \bar{w}_{l}\right)\right|^{-1}=\left(\partial l^{\prime} / \partial \bar{w}_{l}\right)_{j^{\prime}}^{-1}\left(\partial j^{\prime} / \partial \bar{w}_{j}\right)_{w_{l}}
$$

we can write a term in (3.12) in the form

$$
\begin{aligned}
e^{-2 \Phi}\left|\partial\left(j^{\prime}, l^{\prime}\right) / \partial\left(\bar{w}_{j}, \bar{w}_{l}\right)\right|^{-1} d l^{\prime} & =e^{-2 \Phi}\left|\partial j^{\prime} / \partial \bar{w}_{j}\right|^{-1} d \bar{w}_{l} \\
& =P_{j_{;}^{\prime} ; j}^{J}\left(\bar{w}_{l}\right) d \bar{w}_{l},
\end{aligned}
$$

where

$$
P_{j^{\prime} ; j}^{J}\left(\bar{w}_{l}\right)=\left|\partial j^{\prime} / \partial \bar{w}_{j}\right| \bar{w}_{l} e^{-2 \Phi} .
$$

For the off-diagonal element $\left(i f \neq i^{\prime} f^{\prime}\right)$, retracing the above argument from (3.11)-(3.17) one would use in (3.19) (given later)

$$
P_{j^{\prime} ; j}^{J}\left(\bar{w}_{l}\right)=\left[\left(P_{f_{i} ; j_{i}}^{J_{i}}\left(\bar{w}_{l}\right) P_{j_{f}^{\prime} ; j_{f}}^{J_{f^{\prime}}}\left(\bar{w}_{l}\right)\right]^{1 / 2} .\right.
$$

$P_{f_{i} \cdot f_{i}}^{J_{i}}\left(\bar{w}_{l}\right)$ is the (semiclassical) contribution to the collision probability for $j_{i}-j_{i}^{\prime}$ from the trajectory with a given $j_{i}, l, J_{i}, \bar{w}_{l}, \bar{w}_{j}$, and $E$. By specifying $j_{i}$ and $j_{i}^{\prime}$, the value of $\bar{w}_{j}$ needed to reach $j_{i}^{\prime}$ from $j_{i}$ is automatically specified, although there may be several discrete values of such a $\bar{w}_{j}$, for a given $j_{i}, j_{i}^{\prime}, l, \bar{w}_{l}$, and $E$. The summation over such discrete points is indicated by the $\sum$ in (3.12).

It is useful to introduce a further probability term:

$$
\begin{aligned}
\bar{P}_{j^{\prime} ; j}^{J}\left(\bar{w}_{l}\right)= & \left\{(2 J+1) /(2 l+1)\left[(2 j+1)\left(2 j^{\prime}+1\right)\right]^{1 / 2}\right\} \\
& \times P_{j^{\prime}, j}^{J}\left(\bar{w}_{l}\right)
\end{aligned}
$$


which gives a type of joint probability for finding a given $J$ (there are $2 J+1$ states out of a total of $(2 j+1)(2 l+1) j l$-states before collision and typically $2 j^{\prime}+1 \sim 2 j+1$ ) and for reaching state $j^{\prime}$ from state $j$. Hence, Eq. (3.12) for rotational overlapping lines becomes

$$
\begin{aligned}
\sigma_{i f^{\prime}, i f}= & \left(\pi / k^{2}\right) \int_{0}^{\infty}(2 l+1) d l\left[\delta_{i^{\prime} j_{1}} \delta_{f^{\prime} f}-\sum \int_{0}^{1} d \bar{w}_{l}\right. \\
& \left.\times \int_{|j-l|}^{j+l} d J \bar{P}_{j^{\prime} ; j}^{J}\left(\bar{w}_{l}\right) \exp \left(i \theta_{2}\right) D_{6^{\prime} \delta}^{1}(\alpha \beta \gamma)\right] .
\end{aligned}
$$

For the simple case of a rotating dipole, only the matrix elements of $\{m(\omega)\}$ in Eq. (2.3) for which $j_{i}-j_{f}= \pm 1$ and $j_{i}^{\prime}-j_{f}^{\prime}= \pm 1$ need to be calculated The method outlined there then gives the line shape for this rigid rotating dipole. (Other systems which involve additional elements, namely $j_{i}=j_{f}$ and $j_{i}^{\prime}=j_{f}^{\prime}$, i. e., so-called nonresonant spectra, as in the inversion of ammonia, are described by adapting the formal treatment ${ }^{9 b}$ for those cases.)

As already noted, the preceding treatment also applies to rotational-vibrational spectra of linear molecules, when the appropriate vibrational subscripts are added. The line shape prescription is thereby identical to that mentioned above except that the relaxation cross section now becomes

$$
\begin{aligned}
\sigma_{i^{\prime} f^{\prime}, i f} & =\left(\pi / k^{2}\right) \int_{0}^{\infty}(2 l+1) d l\left[\delta_{1^{\prime}, \delta_{\ell^{\prime} f}}-\sum \int_{0}^{1} d \bar{w}_{l}\right. \\
& \times \int_{|j-l|}^{j+l} d J \bar{P}_{j^{\prime} n^{\prime}: j n}^{J}\left(\bar{w}_{l}\right) D_{\delta^{\prime} \delta}^{\prime}(\alpha \beta \gamma) \exp \left[i\left(\theta_{n}+\theta_{2}\right)\right],
\end{aligned}
$$

where $n$ denotes the mean value of the vibrational quantum numbers $n_{i}$ and $n_{f}$ before and after the $i \rightarrow f$ optical transition; the vibrational term $\theta_{n}$ is given by

$$
\theta_{n}=-2 \pi \bar{w}_{n}^{\prime}\left(n_{i}^{\prime}-n_{f}^{\prime}\right)+2 \pi \bar{w}_{n}\left(n_{i}-n_{f}\right),
$$

where $\bar{w}_{n}$ is the coordinate conjugate to $2 \pi n$. (If there is more than one vibration there is the appropriate sum of $\theta_{n}{ }^{\prime} \mathrm{s}$.) The quantity $\bar{P}_{j^{\prime} n^{\prime} ; j n}^{J}\left(\bar{w}_{l}\right)$ becomes

$$
\begin{aligned}
\bar{P}_{j^{\prime} n^{\prime} ; j n}^{J}\left(\bar{w}_{l}\right)= & \left|\frac{\partial\left(j^{\prime}, n^{\prime}\right)}{\partial\left(\bar{w}_{j}, \bar{w}_{n}\right)}\right|_{\bar{w}_{l}}^{-1} e^{-2 \Phi} \\
& \times \frac{2 J+1}{(2 l+1)\left\lceil(2 j+1)\left(2 j^{\prime}+1\right)\right]^{1 / 2}},
\end{aligned}
$$

which is now the (semiclassical) contribution to the collision probability for the transitions $j-j^{\prime}$ and $n \rightarrow n^{\prime}$ from trajectories having a given initial $\left(l, J, \bar{w}_{l} ; E\right)$, and, essentially, for a joint probability of having a given $J$. The sum $\sum$ in $(3.20)$ is over the particular values of $\bar{w}_{j}$ and $\bar{w}_{n}$ which give the desired $j^{\prime}$ and $n^{\prime}$ for the given initial values of the remaining collisional variables. For off-diagonal elements one would use the counterpart of (3.18) instead of the first two factors in (3.23).
For the case of nonoverlapping pure rotational lines, using a rigid rotor model, the shape of a given rotational absorption line is, for states 1 and 2 ,

$$
\begin{aligned}
I(\omega)= & -\frac{1}{\pi}\left|\left\langle j_{1}\|\mu\| j_{2}\right\rangle\right|^{2} \\
& \times \operatorname{Im}\left[\frac{\rho_{1}}{-|\omega|-\omega_{12}+i\left\langle v \sigma_{12 ; 12}\right\rangle}\right. \\
& \left.+\frac{\rho_{2}}{-|\omega|-\omega_{21}+i\left\langle v \sigma_{21 ; 21}\right\rangle}\right] .
\end{aligned}
$$

If 1 and 2 denote the lower and upper states, respectively, then $E_{2}-E_{1}>0$; hence, we let $\omega_{0}=\omega_{21}$ $=-\omega_{12}$. We may now let $\omega$ be a positive quantity (the minus sign in $-|\omega|$ having already taken account of absorption) and let the shift and width be denoted by $d$ and $w$, respectively, where $w+i d$ $=\left\langle v \sigma_{21 ; 21}\right\rangle$. (The latter equals $\left\langle v \sigma_{12 ; 12} *\right\rangle$.) $\langle\cdots\rangle$ denotes a Maxwell-Boltzmann average over velocities. We then have

$$
\begin{gathered}
I(\omega)=\frac{1}{\pi} \operatorname{Im}\left|\left\langle j_{1}\|\mu\| j_{2}\right\rangle\right|^{2}\left[\frac{\rho_{1}}{\omega-\omega_{0}-d-i w}\right. \\
\left.+\frac{\rho_{2}}{\omega+\omega_{0}+d-i w}\right],
\end{gathered}
$$

where $\omega>0$ and

$$
\begin{aligned}
\sigma_{12 ; 12}=\left(\pi / k^{2}\right) & \int_{0}^{\infty}(2 l+1) d l\left[1-\sum \int_{0}^{\infty} d \bar{w}_{l}\right. \\
& \left.\times \int_{|j-l|}^{j+l} d J \bar{P}_{j ; j}^{J}\left(\bar{w}_{l}\right) \exp \left(i \theta_{2}\right) D_{11}^{1}(\alpha \beta \gamma)\right] .
\end{aligned}
$$

The second term in (3.25) is the familiar "negative resonance $"$ term and corresponds to the tail of an absorption centered at $-\left|\omega_{0}\right|{ }^{9 b}$

\section{EXTENSION TO OTHER LINE SHAPE SPECTRA}

The line shape expression (2.1) can be written in a form appropriate to certain additional spectra ${ }^{9 a_{2}}$

$$
\begin{aligned}
& I(\omega)=-\frac{1}{\pi} \operatorname{Im} \sum_{i i^{\prime} f f^{\prime}}\left\langle f^{\prime}\left\|X^{\Pi K}\right\| i^{\prime}\right\rangle\left\langle\left\langle i^{\prime} f^{\prime} ;\right.\right. \\
& \left.\left.\Pi K Q\left|\frac{1}{\omega-\mathbf{L}_{0}^{a}-N\{\mathbf{m}(\omega)\}}\right| i f ; \Pi K Q\right\rangle\right\rangle \rho_{i}\left\langle i|| X^{\Pi K}|| f\right\rangle,
\end{aligned}
$$

where the operator describing the interaction of the system with the radiation is described in general by a multipole $K$ th-order tensor operator $X^{\Pi K}$ (for the $2^{K}$-pole interaction with radiation), $Q$ indicates a particular one of $2 K+1$ standard irreducible components of that operator, and $\Pi$ is the parity of that operator. For the electric dipole spectra in the previous sections we had $K=1, \Pi=-1, X^{\Pi K}=\mu$ and, for radiation polarized along a space-fixed $z$ axis, $Q=0$. For depolarized Raman scattering, $K$ $=2$. In the case of application of (4.1) to the inver- 
sion spectrum of the ammonia molecule, $\Pi$ must be taken into account.

Instead of (2.3) we now have $\left\langle\left\langle i^{\prime} f^{\prime} ; \Pi K Q|\{m(\omega)\}| \Pi K Q\right\rangle\right\rangle=-i \int_{0}^{\infty} v \sigma_{i^{\prime} f^{\prime} ; i f} \rho_{v} 4 \pi v^{2} d v$

The matrix element for $\{\mathrm{m}(\omega)\}$ is diagonal in $K$, $Q$, and $\Pi$ for isotropic gases and independent of $Q$, as shown by Ben-Reuven. ${ }^{9}$ Its properties in the presence of applied fields have been discussed by Snider and Sanctuary. ${ }^{8}$ In the present case of a general $K,\{\mathrm{~m}(\omega)\}$ in Eq. (4.1) is again found to be given by the right hand side of (2.3), but now, as shown in Appendix B, the cross section $\sigma_{i \prime f^{\prime}, \text { if }}$ is that for a system of the given $K$. It is independent of $Q$ and is denoted by $\sigma_{i f^{\prime}, i f}^{K}$ :

$$
\begin{aligned}
& \sigma_{i^{\prime} f^{\prime} ; i f}^{K}=\frac{\pi}{k^{2}} \sum_{l l^{\prime} J_{i} J_{f}}(-)^{j_{i}-j_{i}^{2}+l-l^{\prime}}\left(2 J_{i}+1\right)\left(2 J_{f}+1\right)
\end{aligned}
$$

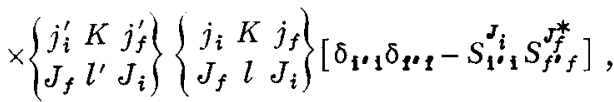

where $i$ in $\sigma$ refers to the appropriate quantum numbers and $i$ consists of this $i, J_{i}$, and $l$. This cross section may be readily expressed semiclassically for rotation-vibration spectra by noting that the only difference between $(2.4)$ and $(4.3)$ is the appearance of a $K$ instead of a 1 . Hence,

$$
\begin{aligned}
\sigma_{i^{\prime} f^{\prime} ; i f}^{K}= & \left(\pi / k^{2}\right) \int_{0}^{\infty}(2 l+1) d l\left[\delta_{i^{\prime} i} \delta_{f^{\prime} f}-\sum \int_{0}^{1} d \bar{w}_{l} \int_{|j-l|}^{j+l} d J\right. \\
& \times \bar{P}_{j^{\prime} n^{\prime} ; j n}^{J}\left(\bar{w}_{l}\right) e^{i\left(\theta_{2^{+}+\theta_{n}{ }^{\prime}} D_{\delta^{\prime} 6}^{K}(\alpha \beta \gamma)\right]}
\end{aligned}
$$

is the cross section.

\section{DISCUSSION}

The diagonal elements in $\sigma_{i \prime f *} ;$ if describe the shift and width of nonoverlapping spectral lines. The off-diagonal elements couple line $i-f$ with line $i^{\prime}-f^{\prime}$ and "transfer intensity" from one to the other, as well as causing collisional narrowing. The effects of such transfers have been discussed by various authors. ${ }^{6,9,11,12}$ The quantum mechanical expression (4.3) for $\sigma_{i, f^{\prime} ; \text { if }}^{K}$ is of the same form as that stated by Gordon, Klemperer, and Steinfeld. ${ }^{13}$

The semiclassical expression for the line shape, given by Eqs. (4. 1), (4.2), and (4.4) is seen, in its "collision cross section" $\sigma_{i f^{\prime} ; i f}^{K}$, to contain several factors: a partial transition probability amplitude $\bar{P}_{j^{\prime} ; j}^{J}$, a rotation matrix element $D^{K}(\alpha \beta \gamma)$, with the angles described in Fig. 2, and an added phase term $\exp i\left(\theta_{2}+\theta_{n}\right)$.

The various quantities appearing in Eq. (4.4) can be evaluated from numerically calculated classical trajectories. It is frequently convenient to do so with the aid of Cartesian coordinates, the choice of initial conditions being made to conform with a given initial $j, l, n, J$, and $E$, as well as with some preassigned $R$ [the relevant results in (3.8) to (3.10) are independent of $R], w_{j}, w_{l}$, and $w_{n}$. The procedure for transforming from Cartesian coordinates to action-angle variables is available in the standard texts. ${ }^{27}$ In application, one selects trajectories which lead to a particular $j^{\prime}$ and $n^{\prime}$; only several values of $w_{j}$ and $w_{n}$ satisfy this condition. From the final data at some preassigned $R^{\prime}$ (the results are independent of $R^{\prime}$ ) one calculates the various quantities, $\bar{w}_{j}^{\prime}, w_{J}^{\prime}-w_{s}$, etc. In this way the integrand in Eq. (4.4) can be calculated as a function of the variables appearing there, $\bar{w}_{l}, l, J$, for any given line pair $i \rightarrow f, i^{\prime} \rightarrow f^{\prime}$.

It is useful to evaluate the factor $D_{\delta^{\circ}{ }_{6}}^{K}(\alpha \beta \gamma)$ $\times \exp i\left(\theta_{2}+\theta_{n}\right)$ in the integrand of (4.4) for several cases and interpret its phase in terms of a phase shift of the mechanical motion. We consider the diagonal elements if $=i^{\prime} f^{\prime}$ first. The term $D_{\tilde{\delta}^{\prime} 6}^{K}$ is given in Tables $\mathrm{I}$ and II for diagonal and off-diagonal elements, for the cases of $K=1$ and $K=2$, respectively.

From Eq. (3.8) and Table I we have for the $R R^{\prime}$ diagonal element, where $i \rightarrow f$ is an $R$-branch transition and $i^{\prime} \rightarrow f^{\prime}$ is also an $R$-branch transition,

$R R^{\prime}$ element:

phase

$$
\begin{aligned}
{\left[D_{6^{\prime}{ }_{0}}^{K}(\alpha \beta \gamma) e^{i\left(\theta_{2}+\theta_{n}\right)}\right]=} & \alpha \\
& +\left(t^{\prime}-t-R^{\prime} / v^{\prime}+R^{\prime} / v\right) \\
& \times \omega_{i f}+\theta_{n} .
\end{aligned}
$$

For the $P P^{\prime}$ case we have

$P P^{\prime}$ element:

$$
\begin{aligned}
& \text { phase } \\
& \begin{aligned}
{\left[D_{\delta^{\prime} \delta}^{K}(\alpha \beta \gamma) e^{i\left(\theta_{2^{+\theta_{n}}}\right)}\right]=} & -\alpha-\gamma \\
& +\left(t^{\prime}-t-R^{\prime} / v^{\prime}+R / v\right) \\
& \times \omega_{i f}+\theta_{n},
\end{aligned}
\end{aligned}
$$

where $\omega_{i f}$ equals $E_{i}-E_{f}$ and so differs in (5.1) and (5. 2).

The right hand sides of (5.1) and (5.2) can be shown to be related to the collisionally induced phase shift in the vibrational-rotational motion, as follows. The $R$ and $P$ branches of the spectrum

TABLE I. Matrix elements $D_{\delta}^{1} \delta(\alpha \beta \gamma)$ for electric dipole transitions.

\begin{tabular}{lcc}
\hline $\begin{array}{l}(j-1)-j \\
\left(P^{\prime} \text { branch }\right)\end{array}$ & $\cos ^{2}(\beta / 2) e^{-i(\alpha+\gamma)}$ & $\sin ^{2}(\beta / 2) e^{i(\alpha-\gamma)}$ \\
$\begin{array}{l}j-(j-1) \\
\left(R^{\prime} \text { branch }\right)\end{array}$ & $\sin ^{2}(\beta / 2) e^{-i(\alpha-\gamma)}$ & $\cos ^{2}(\beta / 2) e^{i(\alpha+\gamma)}$ \\
\hline \hline
\end{tabular}


TABLE II. Matrix elements $D_{6^{\prime} \sigma}^{2}(\alpha \beta \gamma)$ for depolarized Raman scattering.

\begin{tabular}{lccc}
\hline & $\begin{array}{c}(j-2)-j \\
(O \text { branch })\end{array}$ & $\begin{array}{c}(j-1)-(j-1) \\
(Q \text { branch })\end{array}$ & $\begin{array}{c}j-(j-2) \\
(S \text { branch })\end{array}$ \\
\hline $\begin{array}{l}(j-2)-j \\
\left(O^{\prime} \text { branch }\right)\end{array}$ & $\cos ^{4}(\beta / 2) e^{-2 i(\alpha+\gamma)}$ & $\sqrt{3 / 8} \sin ^{2} \beta e^{-2 i \gamma}$ & $\sin ^{4}(\beta / 2) e^{2 i(\alpha-\gamma)}$ \\
$\begin{array}{l}(j-1)-(j-1) \\
\left(Q^{\prime} \text { branch }\right)\end{array}$ & $\sqrt{3 / 8} \sin ^{2} \beta e^{-2 i \alpha}$ & $\frac{1}{2}\left(3 \cos ^{2} \beta-1\right)$ & $\sqrt{3 / 8} \sin ^{2} \beta e^{2 i \alpha}$ \\
$\begin{array}{l}{[j-(j-2)]} \\
\left(S^{\mathrm{y}} \text { branch }\right)\end{array}$ & $\sin ^{4}(\beta / 2) e^{-2 i(\alpha-\gamma)}$ & $\sqrt{3 / 8} \sin ^{2} \beta e^{2 i \gamma}$ & $\cos ^{4}(\beta / 2) e^{2 i(\alpha+\gamma)}$ \\
\hline
\end{tabular}

arise classically from terms in which some component of the mechanical motion oscillates as $\cos 2 \pi w_{n} \cos 2 \pi w_{j}$, and thereby as a composite of $\cos 2 \pi\left(w_{n}+w_{j}\right)$ and $\cos 2 \pi\left(w_{n}-w_{j}\right)$, respectively. The time dependence of $2 \pi\left(w_{n}+w_{j}\right)$ and $2 \pi\left(w_{n}-w_{j}\right)$ is $\left(\omega_{n}+\omega_{j}\right) t$ and $\left(\omega_{n}-\omega_{j}\right) t$, where the $\omega^{\prime}$ s are angular frequencies, and so give rise to the $R$ and $P$ branches, respectively. We consider the $R$ branch first, and calculate the phase of the dipole $O D^{\prime}$ in Fig. 1 relative to that of $O D$. The rotational phase of $O D^{\prime}$ in Fig, 2 is $2 \pi \bar{w}_{j}^{\prime}$. Hence, by (3.8) the rotational phase of $O D^{\prime}, 2 \pi w_{j}^{\prime}$, is $2 \pi\left(\bar{w}_{j}+R \nu_{j}^{\prime} / v^{\prime}\right)$.

These phases are relative to that of $O N$ in Fig. 1. The rotational phase of $O D^{\prime}$ relative to $O N_{j}$ in Fig. 2 is $2 \pi\left(\bar{w}_{j}^{\prime}+R^{\prime} v_{j}^{\prime} / v^{\prime}\right)-a^{\prime}$, where $a^{\prime}$ is the angle from $O N^{\prime}$ to $O N_{j}$. Thus, introducing the angle $\gamma$ defined in Fig. 2, we have

phase $O D^{\prime}\left(\right.$ relative to $\left.O N_{j}\right)=\gamma+2 \pi R^{\prime} \nu_{j}^{\prime} / v^{\prime}$

( $R^{\prime}$ branch).

A similar argument regarding $O D$ yields

$$
\text { phase } \left.O D \text { (relative to } O N_{j}\right)=-\alpha+2 \pi R \nu_{j} / v
$$

(R branch) . (5.4)

The rotational phase shift $\eta_{r}$ from $O D$ to $O D^{\prime}$ is obtained by subtracting (5.4) from (5.3) and then subtracting the time-evolution term $\omega_{j}\left(t^{\prime}-t\right)$ for the free rotational motion. Noting that $\omega_{j}$ equals $2 \pi \nu_{j}$ and $2 \pi \nu_{j}^{\prime}\left(2 \pi \nu_{j}^{\prime} \cong 2 \pi \nu_{j}\right), \eta_{r}$ is seen to be given by (5. 5).

$\eta_{r}=\alpha+\gamma+\left(t^{\prime}-t-R^{\prime} / v^{\prime}+R / v\right) \omega_{j}\left(R R^{\prime}\right.$ element $)$.

The vibrational phase shift $\eta_{v}$ is, by a similar argument, equal to $2 \pi\left(w_{n}^{\prime}-w_{n}\right)-\omega_{n}\left(t^{\prime}-t\right)$. Thus, using (3.10), with $j$ 's replaced by $n$ 's, we have

$$
\eta_{v}=2 \pi\left(\bar{w}_{n}^{\prime}-\bar{w}_{n}\right)-\left(t^{\prime}-t-R^{\prime} / v^{\prime}+R / v\right) \omega_{n} .
$$

The sum of $\eta_{r}$ and $\eta_{v}$ is seen to agree with the right hand side of $(5.1)$ since $\omega_{i f}$ equals $-\left(\omega_{j}+\omega_{n}\right)$ for an $R$ branch and since $\theta_{n}$ is given by (3.22).

We consider next the $P P^{\prime}$ element. Here, the system behaves mechanically as though it had an angular frequency of $\omega_{n}-\omega_{j}$. The vibrational phase shift is given above by (5.6). The rotational phase shift is different, however. The phase of $O D^{\prime}$ relative to $O N^{\prime}$ is now $-2 \pi\left(\bar{w}_{j}+R v_{j}^{\prime} / v^{\prime}\right)$. Relative to $O N_{j}$, it is seen with the aid of Fig. 2 to be

$$
\text { phase } \left.O D^{\prime} \text { (relative to } O N_{j}\right)=-\gamma-2 \pi R^{\prime} \nu_{j}^{\prime} / v^{\prime}
$$

$$
\left(P^{\prime} \text { branch) }\right. \text {. }
$$

Similarly,

phase $O D\left(\right.$ relative to $\left.O N_{j}\right)=\alpha-2 \pi R \nu_{j} / v$

( $P$ branch) .

Subtraction of (5.8) from (5.7) and addition of the time-evolution term $\omega_{j}\left(t^{\prime}-t\right)$ now yields,

$$
\begin{array}{r}
\eta_{r}=-\alpha-\gamma+\left(t^{\prime}-t-R^{\prime} / v^{\prime}+R / v\right) \omega_{f} \\
\left(P P^{\prime} \text { element }\right),
\end{array}
$$

where we have again set $\nu_{j}^{\prime} \approx \nu_{j}=\omega_{j} / 2 \pi$.

The sum of (5.6) and (5.9) agrees with the right hand side of $(5.2)$, which is thus, like (5.1), the rotational-vibrational phase shift.

We consider next the off-diagonal element $P R^{\prime}$, with $n_{i}=n_{i}^{\prime}$ and $n_{f}=n_{f}^{\prime}$ (i.e., a vibrationally diagonal element). From Table II we have

$P R^{\prime}$ element:

$$
\text { phase }\left[D_{\delta^{\circ} \delta}^{K}(\alpha \beta \gamma) e^{i\left(\theta_{2}+\theta_{n}\right)}\right]=-\alpha+\gamma+\theta_{2}+\theta_{n} \text {. }
$$

However, differences in $E_{i}-E_{f}$ and $E_{i}^{\prime}-E_{f}^{\prime}$ are ignored within the impact approximation. ${ }^{7}$ If we replace them by $\frac{1}{2}\left(\omega_{i f}+\omega_{i^{\prime} f^{\circ}}\right)$, (3.8) yields

$$
\theta_{2} \approx \frac{1}{2}\left(w_{E}-w_{E^{\prime}}\right)\left(\omega_{i f}+\omega_{i^{\prime} f^{\prime}}\right) \text {. }
$$

One then finds,

$$
\text { phase }\left[D_{\delta^{\circ} \delta}^{K}(\alpha \beta \gamma) e^{i\left(\theta_{2} * \theta_{n}^{\prime}\right.}\right] \cong-\alpha+\gamma+\eta_{v},
$$

where $\eta_{v}$ is given by (5.6). One might expect $\alpha \cong \gamma$.

To compare (5.12) with the estimated phase shift arising from rotational-vibrational motion we shall need to introduce a "midpoint" of the collision and shall let it occur at $t=0$. The collision time $t_{c}$ can introduce an uncertainty in locating this "midpoint" but uncertainties of the order of $t_{c}$ are neglected by the impact approximation. ${ }^{7}$ The phase of the dipole $O D^{\prime}$ relative to $O N_{f}$ is again given by (5.3), and one subtracts $\omega_{j} t^{\prime}$ to calculate the rotational phase shift contribution from the midpoint of the collision to the end, i. e., from $t=\mathbf{0}$ to $t^{\prime}$. Thus, this contribution to $\eta_{r}$ is $\gamma+2 \pi R^{\prime} \nu_{j}^{\prime} / v^{\prime}-\omega_{j}^{\prime} t^{\prime}$. The phase of $O D$ at the initial time $t(t<0)$ relative to its phase at the midpoint of the collision when $O D$ "moves on a $P$ branch" is again given by $(5,8)$. Upon adding $\omega_{j} t$, we obtain the phase shift of $O D$ for this initial half of the collision, $\alpha-\left(2 \pi R \nu_{j} / v\right)+\omega_{j} t$. Upon subtraction of this quantity for $O D$ from that for $O D^{\prime}$ we obtain $\eta_{r}$ :

$$
\eta_{r}=\gamma-\alpha-\left(t^{\prime}+t-R^{\prime} / v^{\prime}-R / v\right) \omega_{j} .
$$

The vibrational contribution $\eta_{v}$ is again given by 
(5.6). One expects that $\left(t^{\prime}-R^{\prime} / v^{\prime}\right)$ and $t-R / v$ will approximately cancel (e.g., as discussed later) and so $\eta_{r}+\eta_{v}$ agrees with the right hand side of (5.13). Similar remarks apply to the phase of the $R P^{\prime}$ term. These results regarding the $R R^{\prime}, P P^{\prime}, P R^{\prime}$, and $R P^{\prime}$ terms also extend as well as to the various terms arising in the $K=2$ case in Table II.

The phase shift terms of both halves of the collision tend to reinforce in a sense in the $R R^{\prime}$ case, as well as in the $P P^{\prime}$ one. For example, taking the midpoint of the collision to occur at $t=0$, the difference $t^{\prime}-R^{\prime} / v^{\prime}$ for a "hard sphere" collision is negative and equals, in magnitude, the time required to traverse a distance $\sigma$, the hard sphere collision diameter. Similarly, the additional term $-(t-R / v)$, with $t$ and $v$ both negative, equals the same time increment, both in magnitude and in sign, the two terms thus reinforce each other in their sum $t^{\prime}-t-R^{\prime} / v^{\prime}$ $+R / v$. They cancel in the $P R^{\prime}$ and $R P^{\prime}$ terms.

The present results may be compared with the nonperturbative classical results of Gordon ${ }^{11}$ : His Monte Carlo expression for a transition probability is replaced, in our case, by a semiclassical value given by Eq. (3.16). However, we have a partial probability term, a function of $\bar{w}_{l}$, rather than a total probability term. His rotational angle between the $\mathbf{j}$ and $\mathbf{j}^{\prime}$ (our $\beta$ ), and his classical phase shift for the diagonal elements are replaced by our semiclassical ones. He and others have reported that his expression for the line shift is unreliable. ${ }^{12}$ Whether ours will remedy this difficulty will be one of the points we will test in a future numerical application of the present paper. The phase shift for the off-diagonal elements in Ref. 11 does not have the cancellation behavior indicated above.

A virtue of semiclassical theory, aside from the fact that it proceeds directly from the quantum mechanical result, is that it can define phase shifts of trajectories in a precise manner (for the diagonal elements $i f=i^{\prime} f^{\prime}$ at least). For example, in a transition $j-j^{\prime}$, the $j^{\prime}(=j)$ is exactly fixed by singling out certain specific trajectories, while purely classical calculations utilize all possible $j$ "s located within a "box" of $j \pm \frac{1}{2} h$. On the other hand, purely classical calculations have a virtue of considerable simplicity.

Depending on the relative accuracies of the various methods available (exact quantum, classical path, classical, and semiclassical) one may expect to draw upon each of them in future calculations.

Note added in proof. The limits on the semiclassical quantity $J+\frac{1}{2}$ can be shown to be $|l-j|$ and $j+l+1$, and so the limits on $J$ in the integral in Eqs. (3.20), (3.21), (3.26), and (4.4) should read $|l-j|-\frac{1}{2}$ and $l+j+\frac{1}{2}$, instead of $|l-j|$ and $l+j$.

\section{APPENDIX A: SUMMARY OF LIOUVILLE SPACE NOTATION}

Liouville space is a Hilbert space whose vectors are operators in the usual Hilbert space $\mathfrak{H}$ of bra and ket vectors. ${ }^{8,20}$ As such, its vectors are elements of a product space $\mathfrak{H C} \times \mathfrak{H}^{\dagger},|a\rangle\langle b|$,

$$
\left.|a\rangle\langle b|\equiv| a\rangle(|b\rangle)^{\dagger} \equiv|a b\rangle\right\rangle,
$$

which introduces the notation |\rangle$\rangle$ of Baranger. ${ }^{2 b}$ An element of the dual space is $(|a\rangle\langle b|)^{\dagger}$, i.e., $|b\rangle\langle a|$, and can be written as

$$
|b\rangle\langle a| \equiv(\langle b|)^{\dagger}\langle a| \equiv\langle\langle a b| \text {. }
$$

If $|a\rangle\langle b|$ and $\left|a^{\prime \prime}\right\rangle\left\langle b^{\prime \prime}\right|$ are elements, then their usual product, $|a\rangle\left\langle b \mid a^{\prime \prime}\right\rangle\left\langle b^{\prime \prime}\right|$, is also an element.

The scalar product in this Liouville space can be defined by the customary product space definition,

$$
\begin{aligned}
\left\langle\left\langle a^{\prime} b^{\prime} \mid a b\right\rangle\right\rangle & =\left\langle a^{\prime}\right|\left(\left\langle b^{\prime}\right|\right)^{\dagger}|a\rangle(|b\rangle)^{\dagger}=\left\langle a^{\prime} \mid a\right\rangle\left\langle b^{\prime} \mid b\right\rangle^{\dagger} \\
& =\left\langle a^{\prime} \mid a\right\rangle\left\langle b \mid b^{\prime}\right\rangle,
\end{aligned}
$$

or by the customary trace of operators, both definitions yielding the same final result:

$$
\langle\langle C \mid D\rangle\rangle=\operatorname{Tr} C^{\dagger} D,
$$

where $C$ and $D$ denote elements $\left|a^{\prime}\right\rangle\left\langle b^{\prime}\right|$ and $|a\rangle\langle b|$ e.g.,

$$
\left\langle\left\langle a^{\prime} b^{\prime} \mid a b\right\rangle\right\rangle=\operatorname{Tr}\left(\left|b^{\prime}\right\rangle\left\langle a^{\prime}\right|\right)(|a\rangle\langle b|)=\left\langle a^{\prime} \mid a\right\rangle\left\langle b \mid b^{\prime}\right\rangle .
$$

The scalar product $\langle\langle\mid\rangle\rangle$, thus defined, has the usual properties of scalar products.

An operator $\hat{A}$ in Liouville space is the direct product of operators in $\mathfrak{H C} \times \mathfrak{H}^{\dagger}$, e.g., $A_{1} \times A_{2}^{\dagger}$,

$$
\hat{A}|a b\rangle\rangle=\left(A_{1}|a\rangle\right)\left(A_{2}|b\rangle\right)^{\dagger} .
$$

The product of $\hat{A}$ with a scalar, and the sum of two $\hat{A}$ 's is also an operator in this space. Thus, the Liouville operator $\hat{L}$

$$
\hat{L}=[H, I]=H \times I-I \times H
$$

is an operator in this space, $I$ being the identity operator in $\mathcal{H}$ (and in $\mathcal{H}^{\dagger}$ ). The product of operators $\widehat{A}$ and $\hat{B}$ can also be defined in this space.

From the above rules, the matrix element of the above operator $\hat{A}$ is

$$
\left\langle\left\langle\boldsymbol{a}^{\prime} \boldsymbol{b}^{\prime}\left|A_{1} \times A_{2}^{\dagger}\right| a b\right\rangle\right\rangle=\left\langle a^{\prime}\left|A_{1}\right| a\right\rangle\left\langle b\left|A_{2}^{\dagger}\right| b^{\prime}\right\rangle .
$$

Frequently, a star notation has been used, ${ }^{8}$ instead of the $\times$ notation, so that $A_{1} \times A_{2}^{\dagger}$ is written as $A_{1} A_{2}^{*}$, the star indicating that $A_{2}^{*}$ operates on the left hand element,

$$
\begin{aligned}
\left\langle\left\langle a^{\prime} b^{\prime}\left|A_{1} A_{2}^{*}\right| a b\right\rangle\right\rangle & =\operatorname{Tr}\left(A_{2}^{\dagger}\left|b^{\prime}\right\rangle\left\langle a^{\prime}\right|\right)\left(A_{1}|a\rangle\langle b|\right) \\
& =\left\langle b\left|A_{2}^{\dagger}\right| b^{\prime}\right\rangle\left\langle a^{\prime}\left|A_{1}\right| a\right\rangle \\
& =\left(A_{1}\right)_{a^{\prime} a}\left(A_{2}\right)_{b^{\prime} b}^{*} \equiv \hat{A}_{a^{\prime} b^{\prime}, a b} \quad \text { (A8) }
\end{aligned}
$$

yielding the same result as in (A7). With this notation $\hat{L}$ would be $H I^{*}-I H^{*}$. 
It also follows from the above rules that the matrix element of an operator $\hat{A}$ and a vector $\rho$, in this space, $\hat{A}=A_{1} A_{2}^{*}, \quad \rho=\sum_{c d}|c\rangle\langle c|\rho| d\rangle\left\langle d\left|\equiv \sum_{c d}\right| c\right\rangle \rho_{c d}\langle d|$,

is

$$
\left\langle\left\langle a^{\prime} b^{\prime}|\hat{A} \rho| a b\right\rangle\right\rangle=\sum_{c d}\left\langle a^{\prime}\left|A_{1}\right| c\right\rangle\left\langle b\left|A_{2}^{\dagger}\right| b^{\prime}\right\rangle \rho_{c d}\langle d \mid a\rangle .
$$

The Liouville operator $L_{0}^{a}$ related to the rotational-vibrational part of the Hamiltonian of the unperturbed absorber, $H_{0}^{a}$ is ${ }^{8,9}\left[H_{0}^{a},\right]$. If we let $|i\rangle$ and $|f\rangle$ be eigenvectors of the Hamiltonian $H_{0}^{a}$ in ordinary Hilbert space, with eigenvalues $E_{i}^{0}$ and $E_{f}^{0}$, e.g., if $H_{0}^{a}|i\rangle$ equals $E_{i}^{0}|i\rangle$, then $|i\rangle\langle f|$, an operator in ordinary Hilbert space, is an eigenvector of $L_{0}^{a}$ :

$$
\left.\left.L_{0}^{a}|i f\rangle\right\rangle=\left[H_{0}^{a},|i\rangle\langle f|\right]=\omega_{i f}^{0}|i f\rangle\right\rangle,
$$

where $\omega_{i f}^{0}=E_{i}^{0}-E_{f}^{0}$. Thus, the eigenvalues of $L_{0}^{a}$ in this space are the spectral lines, and the space has been called line space. The matrix elements of $L_{0}^{a}$ are seen from (A1) and (A11) to be

$$
\left\langle\left\langle i^{\prime} f^{\prime}\left|L_{0}^{a}\right| i f\right\rangle\right\rangle=\omega_{i f}^{0} \delta_{i^{\prime} i} \delta_{f^{\prime} f} .
$$

If $|i\rangle$ and $|f\rangle$ are normalized, it also follows from the above rules that $|i f\rangle\rangle$ is also normalized, i.e., that $\left\langle\left\langle i^{\prime} f^{\prime} \mid i f\right\rangle\right\rangle$ equals $\delta_{i^{\prime} i} \delta_{f^{\prime} f}$.

The Liouville vector notation in (2.1) and (2.2), where $i$ and $f$ are $j_{i}$ and $j_{f}$, has the following ori$\operatorname{gin}^{9,20}$ : With the usual notation for the rotation operator $R$,

$$
R|j m\rangle=\sum_{m^{\prime}=-j}^{j} D_{m^{\prime} m}^{j}\left|j m^{\prime}\right\rangle
$$

the rotation operator $\hat{R}$ in the product space is defined by

$$
\left.\hat{R}\left|j_{i} m_{i}, j_{f} m_{f}\right\rangle\right\rangle=R\left|j_{i} m_{i}\right\rangle\left(R\left|j_{f} m_{f}\right\rangle\right)^{\dagger}
$$

(i. e., $\hat{R}$ is $R R^{*}$ ), where $\left.\left|j_{i} m_{i}, j_{f} m_{f}\right\rangle\right\rangle$ is $\left|j_{i} m_{i}\right\rangle$ $\times\left\langle j_{f} m_{f}\right|$. One can show that the following Liouville space vector, defined by the right hand side,

$$
\begin{aligned}
& \left.\left|\pi_{i} j_{i}, j_{f} \pi_{f} ; \Pi K Q\right\rangle\right\rangle=\sum_{m_{i} m_{f}}(-1)^{j_{i}-m_{i}} \sqrt{2 K+1} \\
& \left.\times\left(\begin{array}{ccr}
j_{i} & j_{f} & K \\
m_{i} & -m_{f} & -Q
\end{array}\right)\left|\pi_{i} j_{i} m_{i}, \pi_{f} j_{f} m_{f}\right\rangle\right\rangle
\end{aligned}
$$

transforms as

$$
\left.\left.\hat{R}\left|j_{i} j_{f} ; \Pi K Q\right\rangle\right\rangle=\sum_{Q^{\prime}} D_{Q^{\circ} Q}^{K}\left|j_{i} j_{f} ; \Pi K Q^{\prime}\right\rangle\right\rangle .
$$

In (A14) $m_{i}$ and $m_{f}$ are the space-fixed $z$ components of $j_{i}$ and $j_{f}$.

The reduced matrix element is ${ }^{28}$

$$
\begin{aligned}
& \left\langle j_{i}\left\|X^{\Pi K}\right\| j_{f}\right\rangle=\sum_{m_{i} m_{f}}(-1)^{j_{i}-m_{i}}(2 K+1) \\
& \times\left(\begin{array}{ccr}
j_{i} & j_{f} & K \\
m_{i}-m_{f} & -Q
\end{array}\right)\left\langle j_{i} m_{i}\left|X_{Q}^{\Pi K}\right| j_{f} m_{f}\right\rangle,
\end{aligned}
$$

where $X^{\Pi K}=\mu$ and $K=1, Q=0, \Pi=-1$ for the $z$ com ponent of the electric dipole operator.

\section{APPENDIX B: RELATION OF FANO'S OPERATOR RESULT TO EQ. (2.4)}

The number of translational states in the phase space volume element of $d p_{x} d p_{y} d p_{z} d x d y d z$ is $d p_{x} d p_{y} d p_{z} d x d y d z / h^{3}$, and hence the number per unit volume is $d p_{x} d p_{y} d p_{z} / h^{3}$. The number per unit volume with $\mathbf{p}$ in $(\mathbf{p}, \mathbf{p}+d \mathbf{p})$ is thus $p^{2} d p d \hat{\mathbf{p}} / h^{3}$, i.e., $k^{2} d k d \hat{k} /(2 \pi)^{3}$, where $d \hat{p}$ and $d \hat{k}$ denote an infinitesimal solid angle, describing the orientation of $\mathbf{p}$ and of $\mathbf{k}$, respectively. Since the number of translational states per unit volume appears in Fano's $N\{m(\omega)\}$, we thus have

$$
\sum_{\mathbf{k}}=\left[1 /(2 \pi)^{3}\right] \int k^{2} d k d \hat{k} .
$$

If $|\mathbf{k}\rangle$ denotes the plane wave whose coordinate representative $\langle\boldsymbol{r} \mid \mathbf{k}\rangle$ is

$$
\langle\mathbf{r} \mid \mathbf{k}\rangle=\exp i \mathbf{k} \cdot \mathbf{r},
$$

then $|\mathbf{k}\rangle$ can be decomposed $\mathrm{as}^{29}$

$$
|\mathbf{k}\rangle \equiv|k \hat{\mathbf{k}}\rangle=\sum_{l, m}|k l m\rangle\langle k l m \mid k \hat{\mathbf{k}}\rangle,
$$

where

$$
\begin{aligned}
& \langle\mathbf{r} \mid k l m\rangle=c_{k} j_{l}(k r) Y_{l m}(\hat{\mathbf{r}}), \\
& \langle k l m \mid k \hat{\mathbf{k}}\rangle=\left(4 \pi i^{l} / c^{k}\right) Y_{l_{m}}^{*}(\hat{\mathbf{k}}) .
\end{aligned}
$$

$j_{l}$ and $Y_{l_{m}}$ are the spherical Bessel function and spherical harmonic, respectively. The $c_{k}$ 's are chosen so that the $|k l m\rangle$ 's have the normalization

$$
\left\langle k^{\prime} l^{\prime} m^{\prime} \mid k l m\right\rangle=\delta_{l l^{\prime}} \delta_{m m^{\prime}} \delta\left(E-E^{\prime}\right) \text {. }
$$

Using the normalizations of $j_{l}$ and $Y_{l m}$ one finds (on setting $\bar{\hbar}=1$ )

$$
c_{k}=(2 k \mu / \pi)^{1 / 2} \text {. }
$$

It will be recalled from (2.2) that we shall need

$$
\{\mathbf{m}(\omega)\}=\sum_{\mathbf{k} \mathbf{k}^{\prime}} \rho(\mathbf{k})\left\langle\left\langle\mathbf{k}^{\prime} \mathbf{k}^{\prime}|\mathbf{m}(\omega)| \mathbf{k} \mathbf{k}\right\rangle\right\rangle,
$$

where we have used $\mathbf{k}=\zeta$ and where the density operator $\rho$ for the perturber has diagonal elements which depend only on $k$. The Liouville vectors in (B8) are given with the aid of (B3) by

$$
\left.|\mathbf{k k}\rangle\rangle=\sum_{\substack{l m \\ \bar{m}}}|k l m, k \bar{l} \bar{m}\rangle\right\rangle\langle k l m \mid k \hat{\mathbf{k}}\rangle\langle k \hat{\mathbf{k}} \mid k \bar{l} \bar{m}\rangle .
$$

Further, since 


$$
\int d \hat{\mathbf{k}}\langle k l m \mid k \hat{\mathbf{k}}\rangle\langle k \hat{\mathbf{k}} \mid k \bar{l} \bar{m}\rangle=\delta_{l \bar{l}} \delta_{m \bar{m}}(2 \pi)^{3} / \mu k,
$$

one obtains, on integrating (B8) over $\hat{\mathbf{k}}$ and $\hat{\mathbf{k}}^{\prime}$,

$$
\begin{aligned}
\{\mathbf{m}(\omega)\}= & \sum_{\substack{l m \\
l^{\prime} m^{\prime}}} \int_{0}^{\infty} \rho(\mathbf{k}) k^{2} d k \int_{0}^{\infty} k^{\prime 2} d k^{\prime}(1 / \mu k)\left(1 / \mu k^{\prime}\right) \\
& \times\left\langle\left\langle k^{\prime} l^{\prime} m^{\prime}|m(\omega)| k l m\right\rangle\right\rangle .
\end{aligned}
$$

(For later use in (B19) it is helpful to note that in the $k^{\prime}$ integrand we can write $\left(k^{\prime 2} / \mu k^{\prime}\right) d k^{\prime}$ as $d\left(k^{\prime 2} / 2 \mu\right)$.) The Maxwell-Boltzmann distribution function $\rho(\mathbf{k})$ will have the property

$$
1=\sum_{\mathbf{k}} \rho(\mathbf{k})=\left[1 /(2 \pi)^{3}\right] \iint \rho(\mathbf{k}) k^{2} d k d \hat{\mathbf{k}},
$$

and one finds that

$$
\rho(\mathbf{k})=\left(2 \pi / \mu k_{B} T\right)^{3 / 2} \exp \left(-k^{2} / 2 \mu k_{B} T\right) .
$$

To calculate the matrix elements of $\{m(\omega)\}$ in the uncoupled representation we shall need $\left\langle\left\langle j_{i}^{\prime} m_{i}^{\prime}, j_{f}^{\prime} m_{f}^{\prime}\right.\right.$ $\times\left\{\{m(\omega)\}\left|j_{i} m_{i}, j_{f} m_{f}\right\rangle\right\rangle$. Thus, in virtue of (B8) and (B9), this calculation involves that of $\left\langle\left\langle I^{\prime} F^{\prime}|m(\omega)|\right.\right.$ $\times I F\rangle\rangle$, where

$$
\begin{aligned}
\left.|I\rangle=\left|k l m j_{i} m_{i}\right\rangle ;|I F\rangle\right\rangle & =|I\rangle\langle F| \\
& =\left|k l m, j_{i} m_{i}\right\rangle\left\langle k l m, j_{f} m_{f}\right|,
\end{aligned}
$$

with $\left|k l m, j_{i} m_{i}\right\rangle$ denoting a vector in the joint perturber-absorber space, $|k l m\rangle\left|j_{i} m_{i}\right\rangle$.

The scalar product $\left\langle I^{\prime} \mid I\right\rangle$, found from (B6), is

$$
\left\langle I^{\prime} \mid I\right\rangle=\delta\left(E_{i^{\prime}}-E_{i}\right) \delta_{1^{\prime} \mathbf{1}},
$$

where $E_{i}$ is the energy of the absorber-perturber pair in state $|I\rangle$ and where $\delta_{i^{\prime}{ }_{1}}$ now includes $\delta_{m^{\prime} m}$ and $\delta_{m_{i} m_{i}}$ :

$$
\delta_{1^{\prime},}=\delta_{l^{\prime} t} \delta_{m^{\prime} m} \delta_{j_{i}^{\prime} j_{i}} \delta_{m_{i}^{\prime} m_{i}} .
$$

A matrix element for Fano's expression ${ }^{8}$ for the operator $m(\omega)$ is given by ${ }^{30}$

$$
\begin{aligned}
\left\langle\left\langle I^{\prime} F^{\prime}|m(\omega)| I F\right\rangle\right) \cong & \left\langle I^{\prime}\left|T\left(E_{F}^{\prime}+\omega\right)\right| I\right\rangle\left\langle F \mid F^{\prime}\right\rangle \\
& -\left\langle I^{\prime} \mid I\right\rangle\left\langle F^{\prime}\left|T\left(E_{I}-\omega\right)\right| F\right\rangle * \\
& +\pi i \delta\left(E_{I}^{\prime}-E_{F}-\omega\right)\left\langle I^{\prime}\left|T\left(E_{I}^{\prime}\right)\right| I\right\rangle \\
& \times\left\langle F^{\prime}\left|T\left(E_{F}\right)\right| F\right\rangle * \\
& +\pi i \delta\left(E_{F}^{\prime}-E_{I}+\omega\right)\left\langle I^{\prime}\left|T\left(E_{I}\right)\right| I\right\rangle \\
& \times\left\langle F^{\prime}\left|T\left(E_{F}^{\prime}\right)\right| F\right\rangle *,
\end{aligned}
$$

where $T$ is the usual scattering theory transition operator.

It is useful to consider the diagonal elements ( $\left.i f=i^{\prime} f^{\prime}\right)$ of (B17) first. We then set $\omega=E_{i}-E_{f}$ $=E_{i}^{\prime}-E_{f}^{\prime}$, since a particular line $i \rightarrow f$ is being examined. The first $T$-matrix element in (B17) is now $\left\langle I^{\prime}\left|T\left(E_{I}\right)\right| I\right\rangle$, which may be written as $T_{\mathbf{1}^{\prime} i}\left(E_{i}\right)$. Analogous remarks apply to the other elements.
We may then introduce the standard relation ${ }^{31}$ for on-the-energy-shell elements

$$
S_{\mathbf{1}^{\prime} \mathbf{1}}=\delta_{\mathbf{1}^{\prime} \mathbf{1}}-2 \pi i T_{\mathbf{1}^{\prime} \mathbf{1}} \text {. }
$$

Upon introducing (B18) into (B17), noting the remark after (B11) that $\left(k^{\prime 2} / \mu k^{\prime}\right) d k^{\prime}$ equals $d\left(k^{\prime 2} / 2 \mu\right)$, and using the properties of the $\delta$ function we have

$$
\begin{array}{r}
\int_{0}^{\infty}\left\langle\left\langle I^{\prime} F^{\prime}|m(\omega)| I F\right\rangle\right\rangle d\left(k^{\prime 2} / 2 \mu\right)=(2 \pi i)^{-1}\left[\delta_{1^{\prime}{ }_{1}} \delta_{f^{\prime \prime}}\right. \\
\left.-S_{1^{\prime}{ }_{1}}\left(E_{i}\right) S_{f^{\prime}{ }_{f}}^{*}\left(E_{f}\right)\right],
\end{array}
$$

where the $k$ in $E_{i}$ equals that in $E_{f}$. After the manipulations involved in (B21)-(B25), this equation then yields Eq. (2.4) for the diagonal elements.

For the off-diagonal elements the $T$-matrix elements ${ }^{7}$ in (B17) are actually off-the-energy-shell elements. For example, if $E_{i}$ is written as $E_{i}^{0}+k^{2} /$ $2 \mu$, where $E_{i}^{0}$ is the rotational-vibrational energy of the absorber (emitter), and if $E_{i}^{\prime}$ is written as $E_{i}^{0^{\prime}}+k^{\prime 2} / 2 \mu$, an on-the-energy shell element would have $E_{i}^{0}-E_{i}^{0^{\prime}}$ equal to $\left(k^{\prime 2}-k^{2}\right) / 2 \mu$. Similarly, an on-the-shell requirement would lead to $E_{f}^{0}-E_{f}^{0^{\prime}}$ also equalling $\left(k^{\prime 2}-k^{2}\right) / 2 \mu$, and thus equalling $E_{i}^{0}-E_{i}^{0^{\prime}}$, which cannot, in general, be true. How ever, as Baranger has pointed out previously, ${ }^{7}$ such differences can be neglected within the impact approximation.

If, for this off-diagonal case, one subtracts and adds a $\delta_{i^{\prime} i} / 2 \pi$ term to each $\left\langle I^{\prime}|T| I\right\rangle$ in the last two terms of (B17), and adds and subtracts a $\delta_{f^{\prime} f} / 2 \pi$ to each $\left\langle F^{\prime}|T| F\right\rangle^{*}$ there, then integrates over $d\left(k^{\prime 2} / 2 \mu\right)$ using the remark after (B11), one obtains (neglecting differences between $E_{i}^{\prime}$ and $E_{i}$ and between $E_{f}^{\prime}$ and $E_{f}$ ),

$$
\begin{aligned}
& \int_{0}^{\infty}\left\langle\left\langle I^{\prime} F^{\prime}|m(\omega)| I F\right\rangle\right\rangle_{d\left(k^{\prime 2} / 2 \mu\right)=\delta_{i}{ }^{\prime} \delta_{f^{\prime} f}} \\
&-\frac{1}{2}\left[\delta_{i^{\prime} i}-2 \pi i\left\langle I^{\prime}\left|T\left(E_{F}+\omega\right)\right| I\right\rangle\right] \\
& \times\left[\delta_{f^{\prime} f}-2 \pi i\left\langle F^{\prime}\left|T\left(E_{F}\right)\right| F\right\rangle\right]^{*} \\
&-\frac{1}{2}\left[\delta_{i^{\prime} i}-2 \pi i\left\langle I^{\prime}\left|T\left(E_{F}^{\prime}+\omega\right)\right| I\right\rangle\right] \\
& \times\left[\delta_{f^{\prime} f}-2 \pi i\left\langle F^{\prime}\left|T\left(E_{F}^{\prime}\right)\right| F\right\rangle\right]^{*} .
\end{aligned}
$$

Noting the remark already made regarding off-theshell elements, we have written these terms as in (2.4), within the impact approximation.

Equations (B1) - (B20) apply not only to a $(K=1$, $Q=0)$ case, but to a general $K Q$ case. To obtain a reduced matrix element $\left\langle\left\langle i^{\prime} f^{\prime} ; K^{\prime} Q^{\prime}|\{m(\omega)\}|\right.\right.$ if; $K Q\rangle\rangle$, one may use (A14), (B11), (B14), and (B19) to yield

$$
\begin{aligned}
\left\langle\left\langle i^{\prime} f^{\prime} ; K^{\prime} Q^{\prime}|\{m(\omega)\}| i f ; K Q\right\rangle\right\rangle= & -i \int_{0}^{\infty} \rho_{v} 4 \pi v^{2} d v \\
& \times\left(v \sigma_{i{ }^{\prime} f^{\prime} ; \text { if }}\right),
\end{aligned}
$$

where $4 \pi \rho_{v} v^{2} d v$ is the normalized Maxwell-Boltz- 
mann distribution function of velocities and $\sigma_{i^{\prime} f^{\prime} ; \text {; } f}$ is a cross section defined by

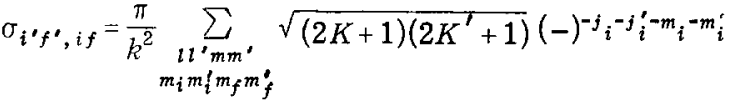

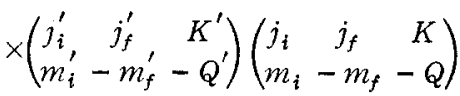

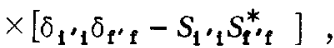

where $\delta_{1} \cdot_{1}$ is given by (B16). Equation (22) may be transformed to the coupled basis by a standard method briefly mentioned below.

The uncoupled states in the above expression may be related to the coupled states by expressions of the type ${ }^{32}$ (omitting $n_{f}$ and $\pi_{f}$ in the bras $\langle 1$, for brevity)

$$
\begin{aligned}
\left\langle j_{f} m_{f} l m\right|= & \sum_{J_{f} M_{f}} \sqrt{2 J_{f}+1}(-1)^{l-j_{f}-M_{f}}\left(\begin{array}{ccc}
j_{f} & l & J_{f} \\
m_{f} & m & -M_{f}
\end{array}\right) \\
& \times\left\langle j_{f} l J_{f} M_{f}\right| .
\end{aligned}
$$

Substitution of expressions like this into (B21), use of the appropriate relationships between the $3-J$ and $6-J$ symbols such as ${ }^{26}$

$$
\begin{array}{r}
\sum_{m_{i} m_{f} m}(-1)^{j_{i}+j_{f}+l+m_{i}+m_{f}+m}\left(\begin{array}{ccc}
j_{f} & l & J_{f} \\
m_{f} & m & -M_{f}
\end{array}\right)\left(\begin{array}{ccc}
j_{i} & l & J_{i} \\
m_{i} & m & -M_{i}
\end{array}\right) \\
\quad \times\left(\begin{array}{ccc}
j_{i} & j_{f} & K \\
m_{i}-m_{f} & -Q
\end{array}\right)=\left(\begin{array}{ccc}
J_{f} & J_{i} & K \\
-M_{f} & M_{i}-Q
\end{array}\right)\left\{\begin{array}{lll}
J_{f} & J_{i} & K \\
j_{i} & j_{f} & l
\end{array}\right\},
\end{array}
$$

and requiring that angular momentum be conserved in the $S$-matrices, yields

$$
\begin{aligned}
& \sigma_{i^{\prime} f^{\prime}, \text { if }}^{K}=\delta_{K K^{\prime}} \delta_{Q Q^{\prime}}\left(\pi / k^{2}\right) \sum_{l l^{\prime} J_{i} J_{f}}(-1)^{j_{i}^{-j_{i}+l-l^{\prime}}} \\
& \times\left(2 J_{i}+1\right)\left(2 J_{f}+1\right)\left\{\begin{array}{lll}
J_{f} & J_{i} & K \\
j_{i} & j_{f} & l
\end{array}\right\}\left\{\begin{array}{lll}
J_{f} & J_{i} & K \\
j_{i}^{\prime} & j_{f}^{\prime} & l^{\prime}
\end{array}\right\}
\end{aligned}
$$

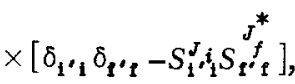

where the subscripts $i$ and $f$ now signify

$$
\mathrm{i} \equiv l j_{i} n_{i}, \quad \mathrm{f} \equiv l j_{f} n_{f} .
$$

It will be noted that $\sigma_{i^{\prime} f^{\prime} ; \text {; } f}^{K}$ is diagonal in $K$ and $Q$ and independent of $Q .{ }^{9}$ The diagonal nature of $\{m(\omega)\}$ in (B21) with respect to parity $\Pi$ arises from the isotropy of the bath of perturbers. ${ }^{9}$

\section{APPENDIX C: PROPERTIES OF THE $F_{4}$ GENERATING FUNCTION AND DERIVATION OF EQ. (3.7)}

We may let the primed variables in (3.4) be the instantaneous values of the variables along the trajectory, rather than merely the final values. The $F_{4}$ is then a generating function for transforming the initial variables to the variables at any point along the trajectory.
There are two $F_{4}$ generating functions which we shall consider, both given by Eq. (3.4) and so numerically equal, but one written as $\bar{F}_{4}\left(\mathrm{n}^{\prime} p_{R}^{\prime} ; \mathrm{n} p_{R}\right)$, and the other written as $F_{4}\left(\mathrm{n}^{\prime} E^{\prime} ; \mathrm{n} E\right)$, where $\mathrm{n}$ denotes the totality of quantum numbers. The properties of $\bar{F}_{4}$ are as follows: The variables canonically conjugate to $2 \pi n_{i}^{\prime}, p_{R}^{\prime}, 2 \pi n_{i}$ and $p_{R}$ are $w_{i}^{\prime}, R^{\prime}$, $w_{i}$ and $R$, where $n_{i}$ and $w_{i}$ denote the $i^{\prime}$ th quantum number and angle variable, respectively. The equations for the transformation from unprimed to primed variables follow from (3.4):

$$
\begin{array}{ll}
w_{i}=\partial \bar{F}_{4} / \partial\left(2 \pi n_{i}\right), & R=\partial \bar{F}_{4} / \partial p_{R}, \\
w_{i}^{\prime}=-\partial \bar{F}_{4} / \partial\left(2 \pi n_{i}^{\prime}\right), & R^{\prime}=-\partial \bar{F}_{4} / \partial p_{R}^{\prime} .
\end{array}
$$

These relations are of the standard form for $F_{4}$ type generating functions. ${ }^{23}$

When instead the generating function $F_{4}\left(\mathrm{n}^{\prime} E^{\prime} ; \mathrm{n} E\right)$ is used, the variables canonically conjugate to $2 \pi n_{i}, E, 2 \pi n_{i}^{\prime}, E^{\prime}$ are denoted by $\vec{w}_{i}, w_{E}, \bar{w}_{i}^{\prime}$, and $w_{E}^{\prime}$, respectively, i.e.,

$$
\begin{array}{ll}
\bar{w}_{i}=\partial F_{4} / \partial\left(2 \pi n_{i}\right), & w_{E}=\partial F_{4} / \partial E \\
\bar{w}_{i}^{\prime}=-\partial F_{4} / \partial\left(2 \pi n_{i}^{\prime}\right), & w_{E}^{\prime}=-\partial F_{4} / \partial E^{\prime} .
\end{array}
$$

These variables have some striking properties: all the $\bar{w}_{i}^{\prime}$ 's and $\bar{w}_{i}^{\prime}$ 's are constants of the motion, while $w_{E}$ and $w_{E}^{\prime}$ are "timelike." These results follow when one observes that $E$ serves as the Hamiltonian for the unprimed variables, while $E^{\prime}$ serves as the Hamiltonian for the primed variables. Thus, using Hamilton's equations of motion, we have

$$
\begin{array}{ll}
\dot{w}_{E}=(\partial E / \partial E)_{n_{i}}=1, & \dot{w}_{E}^{\prime}=\left(\partial E^{\prime} / \partial E^{\prime}\right)_{n_{i}^{\prime}}=1 \\
\vec{w}_{i}=\left[\partial E / \partial\left(2 \pi n_{i}\right)\right]_{E}=0, & \bar{w}_{i}^{\prime}=\left[\partial E^{\prime} / \partial\left(2 \pi n_{i}^{\prime}\right)\right]_{E^{\prime}}=0 .
\end{array}
$$

The relationship of the $\vec{w}^{\prime} \mathrm{s}$ and $w^{\prime} \mathrm{s}$ is seen as

$$
\begin{aligned}
\bar{w}_{i} & =\left(\frac{\partial F_{4}}{\partial\left(2 \pi n_{i}\right)}\right)_{E}=\left(\frac{\partial \bar{F}_{4}}{\partial\left(2 \pi n_{i}\right)}\right)_{D_{R}}+\left(\frac{\partial \bar{F}_{4}}{\partial p_{R}}\right)_{n_{i}}\left(\frac{\partial p_{R}}{\partial\left(2 \pi n_{i}\right)}\right)_{E} \\
& =w_{i}-\frac{R \nu_{i}}{v}, \\
\bar{w}_{i}^{\prime} & =\left(\frac{\partial F_{4}}{\partial\left(2 \pi n_{i}^{\prime}\right)}\right)_{E}=\left(\frac{\partial \bar{F}_{4}}{\partial\left(2 \pi n_{i}^{\prime}\right)}\right)_{D_{R}^{\prime}}+\left(\frac{\partial \bar{F}_{4}}{\partial p_{R}^{\prime}}\right)_{n_{i}} \cdot\left(\frac{\partial p_{R}^{\prime}}{\partial\left(2 \pi n_{i}^{\prime}\right)}\right)_{E} \\
& =w_{i}^{\prime}-\frac{R^{\prime} \nu_{i}^{\prime}}{v^{\prime}},
\end{aligned}
$$

where in the last step of the equation for $\vec{w}_{i}^{\prime}$ we have used the fact that $\vec{w}_{i}$ is a constant and we have evaluated $\partial p_{R}^{\prime} / \partial\left(2 \pi n_{i}^{\prime}\right)$ at the end of the trajectory, so that $w_{i}^{\prime}, R^{\prime}$, and $v^{\prime}$ in (3.10) are the postcollision values now, rather than the values at any point during the collision. Related remarks apply to $\bar{w}_{i}$ in (C5), the last step now being taken at the beginning of the trajectory. The $\nu_{i}$ and $\nu_{i}^{\prime}$ are the pre- and postcollision values of the $i$ th frequency, $\theta H_{0} /$ 
$\partial\left(2 \pi n_{i}^{\prime}\right)$, respectively. Since $H_{0}$ depends on $j$ and $p_{R}$, in Sec. III, but not on $J, \nu_{J}$ is zero, and so we have

$$
\bar{w}_{J}=w_{J}, \quad \bar{w}_{J}^{\prime}=w_{J} .
$$

Equations (C2) and (C5) serve to express the partial derivatives in (3.6) in terms of the various angle variables $\vec{w}_{j}^{\prime}, \bar{w}_{j}, w_{J}^{\prime}, w_{J}, w_{E}$, and $w_{E}^{\prime}$, respectively, which appear in (3.8) and (3.10). Equation (C5) proves (3.10), and there remains now the proof of the last line of (3.8).

Equation (3.4) can be rewritten in terms of the $F_{2}$-generating functions from whence it came, ${ }^{22}$ by an integration by parts

$$
F_{4}\left(n^{\prime} E ; n E^{\prime}\right)=F_{2}\left(w^{\prime} R^{\prime} ; n E\right)-F_{2}^{0}\left(w^{\prime} R^{\prime} ; n^{\prime} E^{\prime}\right),
$$

where

$$
\begin{aligned}
& F_{2}\left(w^{\prime} R^{\prime} ; n E\right)=2 \pi \int_{w}^{w^{\prime}} n d w+\int_{R}^{R^{\prime}} p_{R} d R+2 \pi n w+p_{R} R, \\
& F_{2}^{0}\left(w^{\prime} R^{\prime} ; n^{\prime} E^{\prime}\right)=2 \pi n^{\prime} w^{\prime}+p_{R}^{\prime} R^{\prime},
\end{aligned}
$$

where $n d w$ denotes $\sum n_{i} d w_{i}$, and similar remarks apply to $n w$ and $n^{\prime} w^{\prime}$. We note from (C8) that $2 \pi n^{\prime}$ equals $\partial F_{2} / \partial w^{\prime}$ and $\partial F_{2}^{0} / \partial w^{\prime}$, while $p_{R}^{\prime}$ equals $\partial F_{2} /$ $\partial R^{\prime}$ and $\partial F_{2}^{0} / \partial R^{\prime}$. We recall that $w_{E}$ equals $\partial F_{4} /$ $\partial E$ and so equals $\partial F_{2} / \partial E$, while $w_{E}^{\prime}$ similarly equals $-\partial F_{4} / \partial E^{\prime}$ and $\partial F_{2}^{0} / \partial E^{\prime}$. Thus, at the point $\left(w^{\prime} R^{\prime}\right)$

$w_{E}^{\prime}=\partial F_{2}^{0} / \partial E^{\prime}=\left(\partial F_{2}^{0} / \partial p_{R}^{\prime}\right)\left(\partial p_{R}^{\prime} / \partial E^{\prime}\right)=R^{\prime} / v^{\prime} \quad\left(v^{\prime}>0\right)$.

Again, at the point $\left(w^{\prime} R^{\prime}\right)$

$$
w_{E}=\partial F_{2} / \partial E=w_{E}(\text { at } w R)+t^{\prime}-t,
$$

where $t^{\prime}-t$ is the time for the system to go from $w R$ to $w^{\prime} R^{\prime}$, recalling that $\dot{w}_{E}=1$. However, at the point $w R$, the two integrals in (C8) vanish and so

$$
w_{E}(\text { at } w R)=\partial F_{2} / \partial E(\text { at } w R)=R / v \quad(v<0) .
$$

Equations (C9)-(C11) yield the last line of (3.8).

\section{APPENDIX D}

Conventionally, if three sucessive Euler angle rotations through the angles $\alpha, \beta$, and $\gamma$ are performed (see Fig. 2), the operation may be described by a rotation operator $D(\alpha \beta \gamma)$. Again, performing two successive sets of three Euler angle rotations in the order $\alpha_{1}, \beta_{1}, \gamma_{1}$ followed by $\alpha_{2}, \beta_{2}$, $\gamma_{2}$ can be described by a single composite set of three Euler angle rotations $\alpha, \beta$ and $\gamma$. A convenient relationship between the matrix elements of the rotation operators used to describe these rotations is is $^{33}$

$\sum_{m^{\prime \prime}} D_{m m^{\prime \prime}}^{j}\left(\alpha_{2} \beta_{2} \gamma_{2}\right) D_{m^{\prime \prime} m^{\prime}}^{j}\left(\alpha_{1} \beta_{1} \gamma_{1}\right)=D_{m m^{\prime}}^{j}(\alpha \beta \gamma)$,

where ${ }^{34}$

$$
D_{m m^{\prime}}^{j}(\alpha \beta \gamma)=e^{i m^{\prime} \alpha} d_{m m^{\prime}}^{j}(\beta) e^{i m r}=e^{i m^{\prime} \alpha} d_{m^{\prime} m}^{j}(-\beta) e^{i m \gamma} \text {. }
$$

The procedure used here for executing a set of Euler angle rotations is that in Edmonds. ${ }^{35}$

The right hand side of (3.13) can be rewritten by substituting in the relations for $\theta_{1}$ given by (3.8) and rearranging to give

$$
\begin{aligned}
\sum_{\lambda=-K}^{K} e^{i \theta_{1}} d_{\delta \lambda}^{K}(\xi) d_{\tilde{\sigma}^{\prime} \lambda}^{K}\left(\xi^{\prime}\right)= & \sum_{\lambda=-K}^{K}\left(e^{2 \pi i w_{j}^{\prime} \lambda} d_{\delta^{\prime} \lambda}^{K}\left(\xi^{\prime}\right) e^{2 \pi i \bar{w}_{j}^{\prime} 0^{\prime}}\right) \\
& \times\left(e^{-2 \pi i \bar{w}_{j} 6} d_{\lambda \sigma}^{K}(-\xi) e^{-2 \pi i w_{j} \lambda}\right) .
\end{aligned}
$$

Equations (D1)-(D3) and the right hand side of (3.13) yield

$$
\begin{gathered}
\sum_{\lambda=-K}^{K} D_{\delta^{\prime} \lambda}^{K}\left(2 \pi w_{j}^{\prime}, \xi^{\prime}, 2 \pi \bar{w}_{j}^{\prime}\right) D_{\lambda \sigma}^{K}\left(-2 \pi \bar{w}_{j},-\xi,-2 \pi \bar{w}_{j}\right) \\
=D_{\delta^{\prime} 6}^{K}(\alpha \beta \gamma),
\end{gathered}
$$

where the angles $\alpha, \beta$, and $\gamma$ are shown in Fig. 2 . This result establishes (3.13).

Equations (D1)-(D3) show that the first set of rotations $\left(\alpha_{1} \beta_{1} \gamma_{1}\right)$ in (D1) involves the rotation of $O \bar{D}$ through angles $\left(-2 \pi \bar{w}_{j},-\xi,-2 \pi w_{\mathrm{J}}\right)$, so that $O \bar{D}$ now lies along $O Y$ in Fig. 1 . The second set of rotations $\left(\alpha_{2} \beta_{2} \gamma_{2}\right)$ involves the rotation of this new $O \bar{D}$ into $O \bar{D}^{\prime}$ via the angles $\left(2 \pi w_{J}^{\prime}, \xi^{\prime}, 2 \pi \bar{w}_{j}^{\prime}\right)$. In Fig. 2 we have used $\bar{q}_{j}=2 \pi \bar{w}, \bar{q}_{J}=2 \pi w_{J}$, etc., for clarity in the figure.

*Supported in part by a grant from the National Science Foundation.

'P. W. Anderson, Phys. Rev. 76, 647 (1949).

${ }^{2}$ M. Baranger, Phys. Rev. 111, 494 (1958), which is a formal development using the classical path approximation but avoiding the perturbative approximation for the $S$-matrices.

${ }^{3}$ A. C. Kolb and H. Griem, Phys. Rev. 111, 514 (1958).

${ }^{4}$ J. I. Gersten and H. M. Foley, Phys. Rev. 182, 24 (1969).

${ }^{5}$ H. A. Rabitz and R. G. Gordon, J. Chem. Phys. 53, 1815 (1970); J. Chem. Phys. 52 1831 (1970).

${ }^{6}$ R. G. Gordon and W. B. Neilson, J. Chem. Phys. 58, 4131 (1973); J. Chem. Phys. 58, 4149 (1973).

${ }^{7}$ M. Baranger, Phys. Rev. 112, 855 (1958); cf. Phys. Rev. 111, 481 (1958).

${ }^{8}$ (a) U. Fano, Phys. Rev. 131, 259 (1963); (b) cf. Ref. 5 and R. Zwanzig, Lect. Theor. Phys. 3, 106 (1961); (c) see also R. F. Snide1 and B. C. Sanctuary, J. Chem. Phys. 55, 1555 (1971).

(a) A. Ben-Reuven, Phys. Rev. 141, 34 (1966); (b) Phys. Rev. 145, 7 (1966).

${ }^{10}$ For example, Ref. 5 and references cited therein.

${ }^{11}$ R. G. Gordon, J. Chem. Phys. 44, 3083 (1966); J. Chem. Phys. 45, 1649 (1966).

${ }^{12}$ R. G. Gordon and R. P. McGinnis, J. Chem. Phys. 55, 4898 (1971).

${ }^{13}$ R. G. Gordon, W. Klemperer, and J. I. Steinfeld, Annu. Rev. Phys. Chem. 19, 215 (1968); R. G. Gordon, Adv. Magn. Reson. 3, 1 (1968).

${ }^{14}$ J. J. M. Beenaker, H. F. P. Knaap, and B. C. Sanctuary (to be published)

${ }^{15}$ (a) R. A. Marcus, Chem. Phys. Lett. 7, 525 (1970); (b) J. Chem. Phys. 54, 3965 (1971); (c) J. N. L. Connor and R. A. Marcus, J. Chem. Phys. 55, 5636 (1971); (d) W. H. Wong and R. A. Marcus, J. Chem. Phys. 55, 5663 (1971); (e) J. Chem. Phys. 56, 311 (1972); (f) J. Chem. Phys. 56, 3548 (1972); (g) J. Stine 
and R. A. Marcus, Chem. Phys. Lett. 15, 536 (1972); (h) R. A Marcus, J. Chem. Phys. 57, 4903 (1972); (i) R. A. Marcus, Discuss. Faraday Soc. (to be published); (j) J. N. L. Connor, Mol. Phys. 23, 717 (1972); Discuss. Faraday Soc. (to be published).

${ }^{16}$ W. H. Miller, J. Chem. Phys. 53, 1949 (1970); J. Chem. Phys. 53, 3578 (1970); Chem. Phys. Lett. 7, 431 (1970); J. Chem. Phys. 54, 5386 (1971); W. H. Miller, Acc. Chem. Res. 4, 161 (1971); C. C. Rankin and W. H. Miller, J. Chem. Phys. 55, 3150 (1971); W. H. Miller and T. F. George, J. Chem. Phys. 56, 5668 (1972); T. F. George and W. H. Miller, J. Chem. Phys. 56, 5722 (1972); J. Chem. Phys. 57, 2458 (1972); J. D. Doll and W. H. Miller, J. Chem. Phys. 57, 5019 (1972); J. D. Doll, T. F. George, and W. H. Miller, J. Chem. Phys. 58, 1343 (1973); W. H. Miller and A. W. Raczkowski, Discuss. Faraday Soc. (to be published).

${ }^{17}$ See also related semiclassical studies of P. Pechukas, Phys. Rev. 181, 166 (1969); Phys. Rev. 181, 174 (1969); R. D. Levine and B. R. Johnson, Chem. Phys. Lett. 7, 404 (1970); Chem. Phys. Lett. 8, 501 (1971); I. C. Percival and D. Richards, J. Phys. B 3, 315 (1970); J. Phys. B 3, 1035 (1970).

${ }^{18}$ A. R. Edmonds, Angular Momentum in Quantum Mechanics (Princeton U.P., Princeton, New Jersey, 1960); 2nd ed., Appendix 2, p. 122. To put the 6-j symbols in (2.4) in the form of Edmonds, write

$$
\begin{aligned}
& \left\{\begin{array}{ccc}
J_{f} & J_{i} & K \\
j_{i} & j_{f} & l_{i}
\end{array}\right\}=\left\{\begin{array}{ccc}
J_{i}+m & J_{i} & K \\
j_{i} & j_{i}+\delta & l_{i}
\end{array}\right\}=\left\{\begin{array}{ccc}
l_{i} & j_{i} & J_{i} \\
K & J_{i}+m & j_{i}+\delta
\end{array}\right\} \\
& =\frac{(-1)^{l i+j_{i}+J_{i}}}{\left.\left[\sqrt{\left(2 j_{i}\right.}+1\right)\left(2 J_{i}+1\right)\right]^{1 / 2}} d_{-m 5}^{K}(\pi-\xi)
\end{aligned}
$$

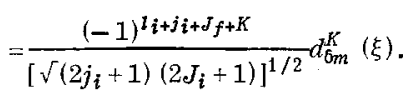

${ }^{19} I(\omega)$ is the relevant collision-dependent factor appearing in
$\operatorname{Im}[\epsilon(0)-\epsilon(\omega)]$ of Eq. (22) in Ref. 9(b). See also Eq. (21) of Re $8(\mathrm{a})$.

${ }^{20}$ J. Fiutak, Zesz. Nauk Wyzsz, Szk. Pedagog. Gdansku: Mat., Fiz., Chem. 8, 39 (1968) (in English); J. Fiutak and J. Van Kranendonk, Can. J. Phys. 40, 1085 (1962).

${ }^{21}$ That is, Ref. 15(d), Footnote 8.

${ }^{22}$ That is, see R. A. Marcus, Ref. 15(h), Eq. (2.9), which is the same as the present (3.3).

${ }^{23}$ H. Goldstein, Classical Mechanics (Addison-Wesley, Reading, MA, 1965), p. 242. We have reversed the order of the symbols in $F_{4}$. (The primed variables are Goldstein's new variables.)

${ }^{24}$ That is, S. Geltman, Topics in Atomic Collision Theory (Academic, New York, 1969), p. 34.

${ }^{25}$ Reference 18 , p. 55.

${ }^{26}$ This relation follows from the properties of determinants and was introduced by J. D. Doll and W. H. Miller, J. Chem. Phys. 57, 5019 (1972) in their "partial averaging" technique.

${ }^{27}$ E. T. Whittaker, A Treatise on Analytical Dynamics (Cambridge U.P., Cambridge, 1927), 3rd., p. 339ff; L. A. Pars, A Treatise on Analytical Dynamics (Wiley, New York, 1965), p. 573ff.

${ }^{28}$ Reference 18 , p. 75.

${ }^{29}$ A. Messiah, Quantum Mechanics (North-Holland, Amsterdam, 1966), Vol. 1, p. 497, Eq. (B105).

${ }^{30}$ See also Eq. (15) of Ref. 9(b) but note that the first two terms there are in error (they each tacitly contain a product $\delta\left(E_{i}^{\prime}-E_{i}\right) \delta\left(E_{f}^{\prime}-E_{f}\right)$, instead of one of these $\delta$ 's above. They should read as in the present Eq. (B17).

${ }^{31}$ Reference 29, Vol. 2, p. 866.

${ }^{32}$ Reference 18 , pp. 46, 95.

${ }^{33}$ D. M. Brink and G. R. Satchler, Angular Momentum (Clarendon, Oxford, 1968), pp. 146, 147. The rotations carried out in this reference are conventionally different from those in Edmonds. Eq. (D1) applies nevertheless.

${ }^{34}$ Reference 18 , pp. 55, 59

${ }^{35}$ Reference 18, pp. 6, 7. 